\title{
ORIGINAL ARTICLE Retinoic acid directs breast cancer cell state changes through regulation of TET2-PKCל pathway
}

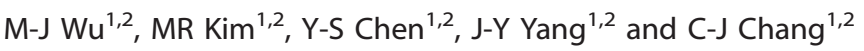

The key molecular mechanism governing the cancer cell state (stem cell-like state vs differentiation state) to control the cancer stem cell (CSC) pool remains elusive. This study provides the first evidence showing that all-trans retinoic acid (ATRA) induces the interaction and chromatin recruitment of a novel RAR $\beta$-TET2 complex to epigenetically activate a specific cohort of gene targets, including MiR-200c. TET2-activated miR-200c further targets and suppresses PKC, a cell polarity protein that has a pivotal role in directing asymmetric division of mammalian stem cells to sustain the stem cell pool. Our data reveal that pharmacological concentration of ATRA effectively downregulates PKC through activation of miR-200c, leading to a decrease of the stem cell-like populations from non-tumorigenic mammary epithelial cells and non-aggressive breast cancer cells. However, aggressive breast cancer cells that manifest TET2-miR-200c dysregulation sustain a CSC pool highly resistant to ATRA, where inhibition of PKCZ directs the resistant CSCs to the luminal cell-like state and sensitization to tamoxifen, resulting in abrogation of mammary tumor growth and progression. Together, these findings elucidate a novel RARß-TET2-miR-200c-PKC signaling pathway that directs cancer cell state changes and also provide previously unidentified therapeutic implications for PKC inhibitors in diminishment of breast CSCs to eradicate breast cancer.

Oncogene (2017) 36, 3193-3206; doi:10.1038/onc.2016.467; published online 20 February 2017

\section{INTRODUCTION}

During tumorigenesis, the generation of cancer stem cells (CSCs), a subset of the cancer cell population that possesses self-renewal properties associated with normal stem cells, allows the perpetual rise of the bulk of a tumor as the 'seed' of the cancer. ${ }^{1-4}$ Accumulated evidence shows that the cell fate decision that favors asymmetric division (AD) of stem cells is critical to ensure a self-renewing and sustaining stem cell pool. ${ }^{5-7}$ It has been known that atypical protein kinase $\mathrm{C}(\mathrm{aPKC})$, the cell polarity protein family that includes $\mathrm{PKC} \zeta$ and $\mathrm{PKCl} / \lambda$, directs self-renewing $A D$ through phosphorylation and asymmetric segregation of the cell fate determinant NUMB (S276/S295) to only one of the progenies to maintain the stem cell pool. ${ }^{6,7}$ In contrast, loss of aPKC expression/activity leads to a uniform distribution of NUMB with the consequent symmetric commitment (SC) of both daughter cells to the differentiation cell state, resulting in decrease of the stem cell pool..$^{5-7}$ Nonetheless, the critical mechanism underlying regulation of aPKC that directs the cell fate decision to govern the cancer cell state changes (stem cell-like state vs differentiation state) still remains to be elucidated.

Numerous studies have shown that RA signaling directs stem cell differentiation and is critical for the maintenance of the stemness differentiation homeostasis. ${ }^{8}$ All-trans retinoic acid (ATRA), a natural derivative of vitamin $A$, is a potent molecule for inducing cell differentiation and the only example of a clinically useful cytodifferentiating agent in treatment of several solid tumor types, including breast cancer, ${ }^{8-12}$ presents an attractive modality of treatment that targets the CSC population and tends to be less toxic and more specific than conventional chemotherapy. ${ }^{8}$ Even through some of the trial patients receive significant therapeutic benefits, a large proportion of patients with aggressive/relapsed breast cancers exhibit only modest/nonsignificant therapeutic effects. ${ }^{8,13}$ Notably, studies have shown that retinoic acid receptors (RARs), a nuclear receptor family that binds to retinoid X receptor (RXRs) as heterodimer (RAR/RXR) to the RAR response element (RAR-RE) in the target gene promoters, undertake conformational changes to recruit transcriptional coactivators or to dissociate co-repressors once the RARs have interacted with the RA ligands, such as ATRA, leading to transcriptional activation of the target genes. ${ }^{13}$ Among the RARs (RARa, RAR $\beta$ and RAR $\gamma$ ), lost RAR $\beta$ expression is most significantly correlated with tumor grade and is also associated with RA resistance. ${ }^{13}$ However, the mechanism that links RAR $\beta$ to RA response (sensitivity vs resistance) is yet to be established.

This study uncovers a novel RARß-TET2-miR-200c-PKCZ signaling pathway that directs breast cancer cell state changes in response to ATRA. The resulting findings provide important molecular insights into new strategies to overcome the therapeutic hurdles of aggressive breast cancer.

\section{RESULTS}

ATRA induces interaction and nuclear colocalization of RAR 3 -TET2 To determine the mechanism(s) underlying RAR $\beta$-regulated ATRA response, a mass spectrometry analysis was used to identify potential chromatin proteins that specifically interact with RAR $\beta$ in response to a pharmacological concentration of ATRA treatment $\left(10^{-6} \mathrm{M}\right)^{8,13}$ in MCF12A, a non-tumorigenic, immortalized mammary epithelial cell line with intact RAR $\beta$ expression and RA response. Interestingly, we found that a methylcytosine dioxygenase, ten eleven translocation2 (TET2), was associated with RAR $\beta$ in ATRA-treated MCF12A cells (Supplementary Table S1,

${ }^{1}$ Department of Basic Medical Sciences, Purdue University, West Lafayette, IN, USA and ${ }^{2}$ Center for Cancer Research, Purdue University, West Lafayette, IN, USA. Correspondence: Dr C-J Chang, Department of Basic Medical Sciences, Purdue University, 625 Harrison Street, West Lafayette, IN 47907, USA.

E-mail: chunjuchang@purdue.edu

Received 13 May 2016; revised 24 October 2016; accepted 8 November 2016; published online 20 February 2017 
TET2 had the highest number of the peptides matched for the protein with the highest sequence coverage among the identified RAR $\beta$-interacting proteins), where endogenous RAR $/ R X R a$ can also be reciprocally co-immunoprecipitated with TET2 in MCF12A cells, as well as in T47D cells, a non-invasive breast cancer cell line (Figure 1a, Supplementary Figure S1). GST pull down data also validated a direct physical interaction between RAR $\beta$ and TET2 proteins in vitro, where their interaction was substantially enhanced in the presence of ATRA (Figure $1 \mathrm{~b}$ ). It is well recognized that the TET protein family has a key role in active DNA demethylation by catalyzing the conversion of the modified genomic base 5-methylcytosine into 5-hydroxymethylcytosine $(5 \mathrm{hmC})$, thereby activating the target gene expression. ${ }^{14}$ Among the three human TET proteins (TET1-TET3), TET2 is the most predominantly expressed in normal breast tissue and the expression is significantly repressed in aggressive breast a

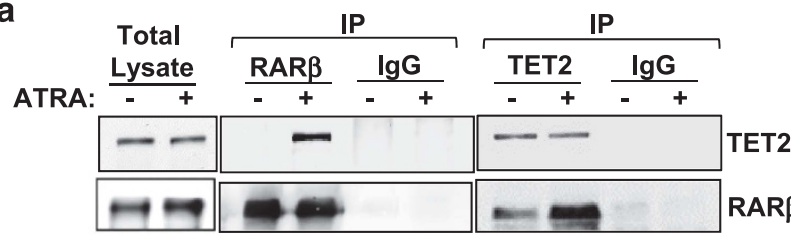

C

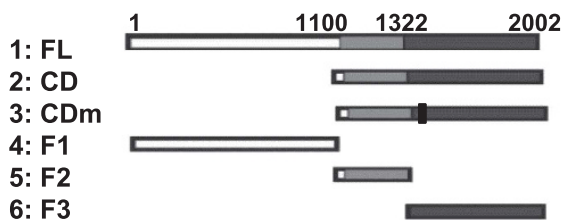

6: F3

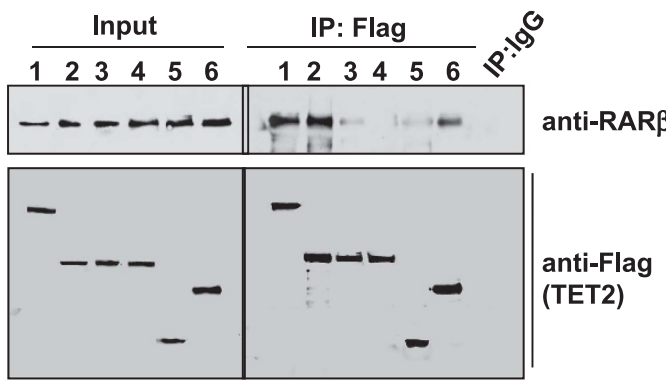

e

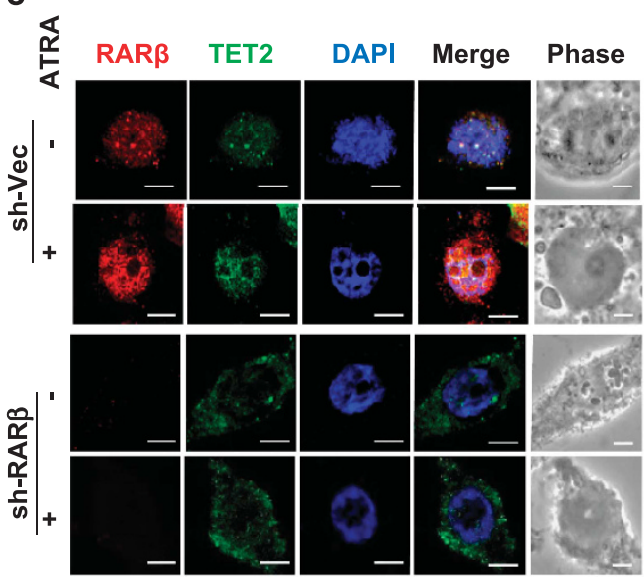

f

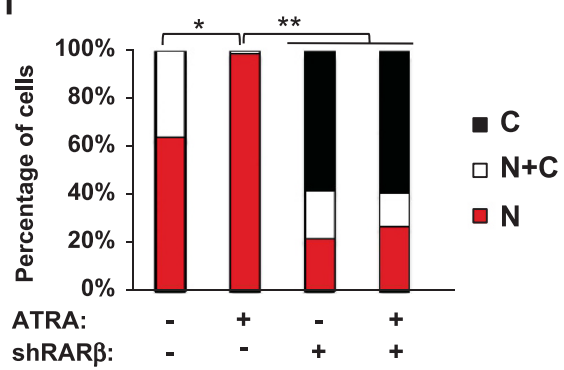

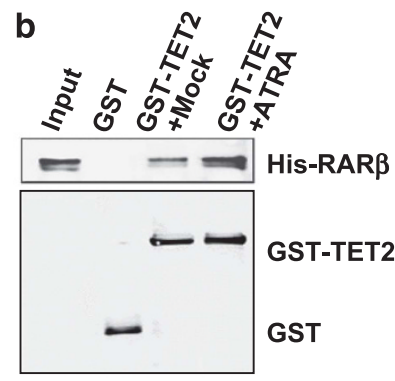

d

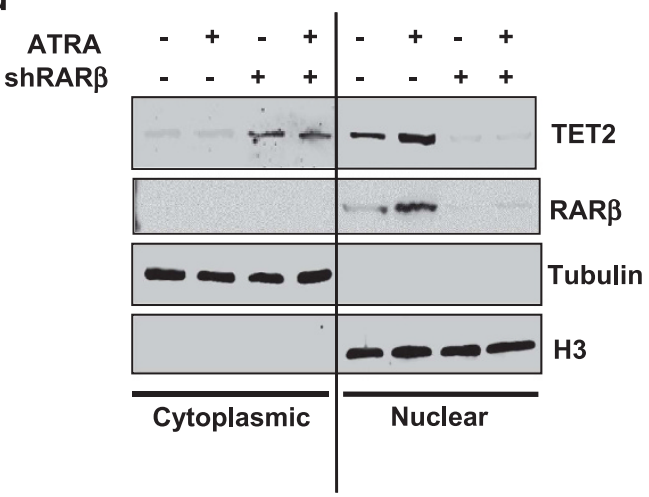

g

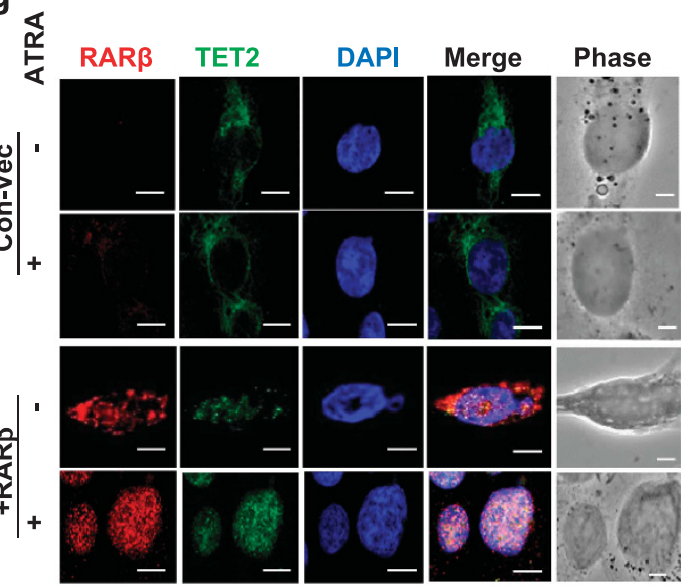

h

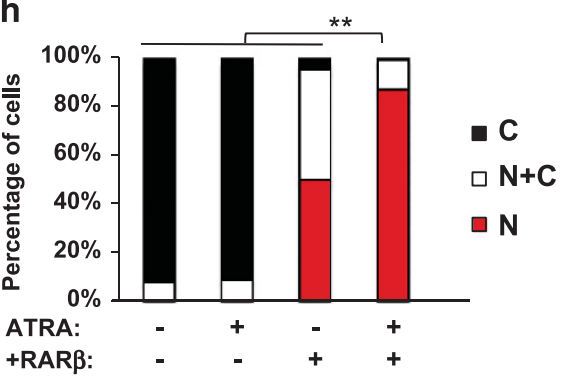


cancers. ${ }^{15}$ To gain functional insight into RAR $\beta-T E T 2$ interaction, we analyzed the interaction of RAR $\beta$ with TET2 mutant proteins along with deletions of specific functional domains ${ }^{16}$ (the domains are conserved between human and mouse). The data showed that an intact C-terminal catalytic domain $(C D$, containing DSBH and cysteine-rich domains) was required for both human and mouse TET2 to have optimal interactions with RAR $\beta$ (Figure 1C, Supplementary Figure S2). Furthermore, it is known that RAR nuclear translocation can be induced by ATRA; ${ }^{13}$ however, the regulatory mechanism underlying nuclear localization and chromatin recruitment of TET2, which has no apparent nuclear localization signal sequence or DNA-binding domain, still remains largely unknown. We found that compared with the mock-treated cells, ATRA enhanced the nuclear localization of RAR $\beta$; interestingly, ATRA also elevated TET2 expression in the nuclear fraction, whereas knockdown of RAR $\beta$ blocked ATRAmediated TET2 nuclear localization and substantially increased TET2 expression in the cytoplasm fraction (Figure 1d). Concordantly, confocal immunofluorescence analysis showed that ATRA significantly increased localization of TET2 to the nucleus, where it colocalized with RAR $\beta$ (Figures 1e and f). However, TET2 relocalized to the cytoplasm upon knockdown of RAR $\beta$ using lentivirus-expressed short hairpin RNA (sh-RAR $\beta$ ) in MCF12A cells (Figures $1 \mathrm{e}$ and $\mathrm{f}$ ). These results could be recapitulated in a luminal breast cancer cell line, T47D, where RAR $\beta$ expression is required for increased TET2 nuclear localization along with an enhanced $5 \mathrm{hmc}$ level by ATRA treatment (Supplementary Figure S3).

In contrast to MCF12A and T47D cells, TET2 was predominantly localized in the cytoplasm in aggressive triple-negative breast cancer (TNBC) cell line MDA-MB-231, which is deficient in endogenous RAR $\beta$ expression (Figures $1 \mathrm{~g}$ and $\mathrm{h}$ ). Re-expression of RAR $\beta$ in MDA-MB-231 cells re-localized TET2 to the nucleus, and the nuclear TET2 level was further enhanced by ATRA treatment (Figures $1 \mathrm{~g}$ and $\mathrm{h}$ ). Next, we ectopically expressed the TET2 fulllength (FL) and mutant proteins (NT, N-terminal domain, and $C D$, catalytic domain, Figure 1c) along with wild-type RAR $\beta$ in the MDA-MB-231 cells. We found that the FL- and CD-TET2 proteins, which showed strong interaction with RAR $\beta$ in Figure $1 c$, were localized to the cytoplasm in the absence of RAR 3 . FL-and CDTET2 started to exhibit nuclear localization upon ectopic expression of RAR $\beta$, and their nuclear localization was further enhanced by ATRA, along with the increased nuclear RAR $\beta$ (Supplementary Figure S4). In contrast to FL-and CD-TET2, NT-TET2, which could not interact with RAR $\beta$ (Figure 1c), remained in the cytoplasm and did not respond to ATRA nor to the ectopic expression of RAR $\beta$ (Supplementary Figure S4).

ATRA-induced RARß-TET2 complex co-occupies targeted promoters of the genes involved in cell differentiation

To understand whether and how the interaction of RAR $\beta$ and TET2 impacts global gene expression, we performed genome-wide chromatin immunoprecipitation sequencing (ChIP-seq) analysis to identify the RAR $\beta$ and TET 2 bound chromatin regions. We found that ATRA most significantly enhanced RAR $\beta$-TET2 co-occupancy in promoters of a cohort of genes involved in cell differentiation (Figures 2a and b), such as RUNX1, BMP6, IKZF1 and CAV1, by gene ontology enrichment analysis (Supplementary Table S2, false discovery rate $<0.5, P<0.01$ ). Furthermore, RAR $\beta$-TET2 also cooccupied promoters of several non-coding RNAs, such as MicroRNA-200c-3p (MiR-200c-3p) and MicroRNA-205-5p (MiR-205$5 p$; representative MiR-200c peak graph shown in Figure $2 \mathrm{c}$ ), both of which are known to regulate stemness properties. ${ }^{17-20}$ Peak and motif analysis revealed that the majority of RAR $\beta$-TET2 peaks were located at promoters/transcription start sites of high CpG (HCP) content, where putative RAR-REs-AGGTCAnnnnnAGGTCAare located right next to $\mathrm{CpG}$ islands that were strongly associated with $5 \mathrm{hmc}$ (Figures 2c-f: the gray bar denotes RAR-RE; the black bar denotes CPG island, $P<0.001)$. Consistently, the expression levels of RAR $\beta$-TET2 targeted genes/miRNAs were significantly upregulated by ATRA treatment, among which miR-200c was the most activated (Figure $2 \mathrm{~g}$ ). Furthermore, the gene/miRNA expression levels increased by ATRA were abolished upon knockdown of RAR $\beta$ or TET2 (sh-RAR $\beta$, sh-TET2) in MCF12A cells (Figures $2 \mathrm{~g}$ and h), where knocking-down RAR $\beta$ also markedly diminished the nuclear TET2 level (Figure 2h). Together, these data suggest that ATRA induces physical association and chromatin recruitment of the RAR $\beta$-TET2 complex to specific promoter regions that contain RAR-REs next to CpG islands, which can be critical for ATRAmediated activation of the target genes involved in regulation of cell differentiation stemness homeostasis.

ATRA activates miR-200c expression via recruitment of RAR $\beta$-TET2 to a specific MiR-200c promoter region and mediates conversion of 5-methylcytosine to $5 \mathrm{hmc}$

Following the ChIP-seq data, we analyzed the changes in the global miRNA expression profile in response to the ATRA treatment using a genome-wide miRNA-PCR array consisting of 1066 annotated miRNAs, and we found that ATRA significantly upregulated a subset of microRNAs in MCF12A cells, among which microRNA-200c-3p (miR-200c) was the most significantly upregulated (Supplementary Figure S5a, $>2.5$-fold increase compared with the mock treatment, $P<0.01$ ). Despite the previous finding that links TET proteins to activated miR-200c expression, ${ }^{15}$ the regulatory mechanism underlying TET-mediated activation of miR-200c expression is largely unclear.

Therefore, to investigate whether ATRA-RAR $\beta$ indeed has a role in regulation of TET2-mediated activation of miR-200c, we first analyzed, using promoter analysis (Genomatix MetInspector, Ann Arbor, MI, USA), the transcription factor response elements located within the 2-kb region upstream of the transcription starting site of MiR-200c. We found eight putative RAR response elements (RAR-RE, s1-s8) located within the MiR-200c promoter that had

Figure 1. ATRA induces interaction of RAR $\beta$-TET2 and nuclear localization of TET2. (a) Reciprocal co-immunoprecipitation assay showing interaction between endogenous RAR $\beta$ and TET2 in MCF12A cells treated with $10^{-6} \mathrm{M}$ ATRA or vehicle for $6 \mathrm{~h}$ (input: $10 \%$ total cell lysate). (b) GST pull down assay showing direct association of His-RAR $\beta$ and GST-TET2 recombinant proteins in vitro. (c) Full-length (FL) and deletion mutants of Flag-tagged human TET2 were expressed and immunoprecipitated with the Flag antibody followed by immunoblotting for RAR $\beta$ in MCF12A cells treated with $10^{-6} \mathrm{M}$ ATRA or vehicle for $6 \mathrm{~h}$. The TET2 mutants include the C-terminal catalytic domain (CD, containing DSBH and cysteine-rich domains), CD domain carrying H1382Y/D1384A mutation (CDm), N-terminal domain (NT), cysteine-rich domain (F1) and DSBH (double-strand beta helix) domain (F2). (d) Protein levels in the nuclear and cytoplasmic fractions of MCF12A cell lysates that stably expressed the control vector or sh-RAR $\beta$ and treated with $10^{-6} \mathrm{M}$ ATRA or vehicle for $6 \mathrm{~h}$. (e) Confocal immunofluorescence staining images showing subcellular localization of RAR $\beta$ and TET2 in MCF12A cells that expressed the control vector (sh-Vec) or sh-RAR $\beta$ and treated with $10^{-6} \mathrm{M}$ ATRA or vehicle for $6 \mathrm{~h}$. (f) Bar graphs showing the percentage of cells with TET2 localized to the nucleus (N), cytoplasm (C) or both (N $+C)$ analyzed from the images in e. (g) Confocal immunofluorescence staining images showing subcellular localization of RAR $\beta$ and TET2 in MDA-MB-231 cells that expressed the control vector (Con-Vec) or RAR $\beta$ expression plasmid and treated with $10^{-6} \mathrm{M}$ ATRA or vehicle for $6 \mathrm{~h}$. (h) Bar graphs showing the percentage of cells with TET2 localized to the nucleus (N), cytoplasm (C) or both (N+C) analyzed from the images in g. In all, 700-1000 cells were counted per sample from three independent experiments, asterisk indicates $P<0.05$, double asterisk indicates $P<0.01$. Comparable amount of control vector and vehicle were included as negative control (-) in each group. Error bars denote \pm s.d. 

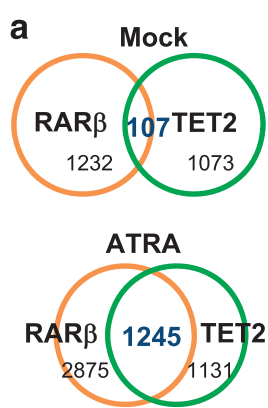

b

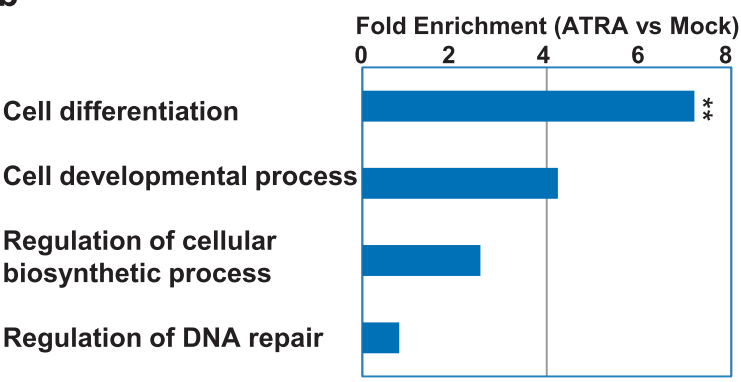

c

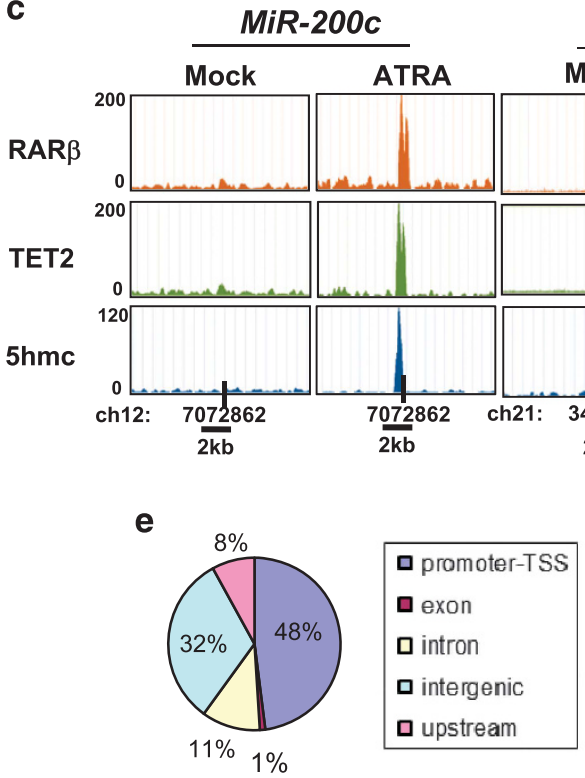

RUNX1 Mock ATRA
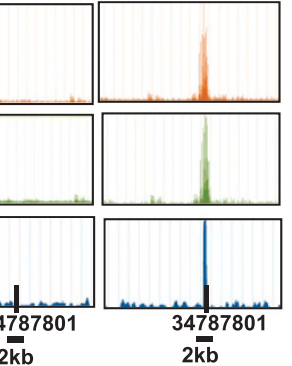

d

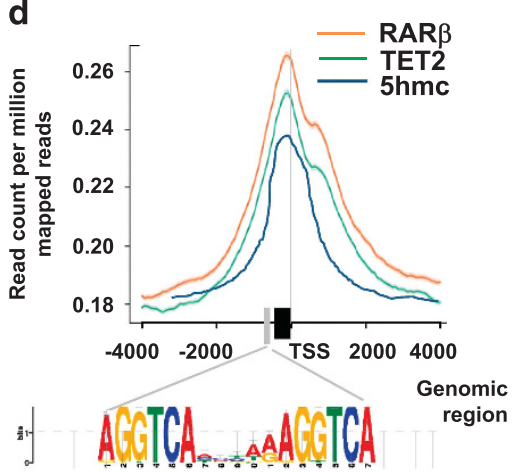

f
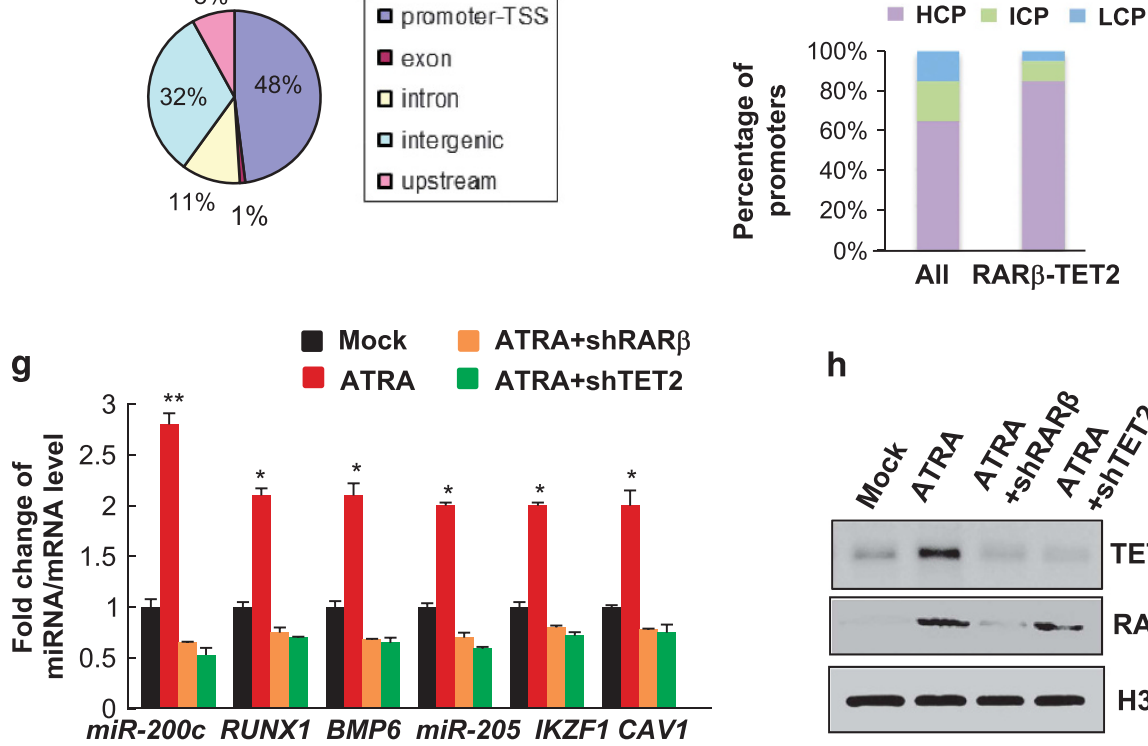

$\mathbf{h}$

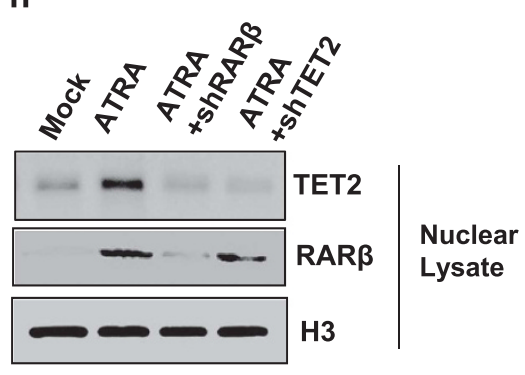

Figure 2. ATRA-induced RAR $\beta$-TET2 co-occupancy in targeted promoters of genes involved in cell differentiation. (a-f) ChIP-sequencing analysis results of MCF12A cells treated with $10^{-6}$ M ATRA or vehicle for $12 \mathrm{~h}(n=3$ independent experiments, double asterisk indicates $P<0.01$ ): (a) Venn diagram showing overlap of RAR $\beta$ and TET2-bound genes, (b) gene ontology functional annotation of RAR $\beta$ and TET2bound genes showing enrichment in specific biological processes, (c) peak histograms showing RAR $\beta$ and TET2 co-occupied MiR-200c (left) and RUNX1 (right) promoters with enhanced 5hmc (bar positions denote the transcription start site (TSS)), (d) RAR $\beta$, TET2, and 5hmc peak distribution around TSS (gray bar denotes RARE motif, black bar denotes CpG island), (e) pie chart showing the percentage of RAR $\beta$ and TET2 co-occupied genomic regions that belong to promoter-TSS, exon, intron, intergenic or upstream regions, and (f) histograms showing the percentage of gene promoters that are divided into high-, intermediate- or low CpG density promoters (HCP, ICP or LCP) for all genes and for RAR $\beta$-TET2 co-occupied genes. (g) Fold change of miRNA/mRNA expression, and (h) nuclear protein expression levels in MCF12A cells that stably expressed sh-RAR $\beta$ or sh-TET2 and treated with $10^{-6}$ M ATRA or vehicle for $12 \mathrm{~h}(n=3$ independent experiments, asterisk indicates $P<0.05$, double asterisk indicates $P<0.01)$. Comparable amount of control vector and vehicle were included as negative control $(-)$ in each group. Error bars denote \pm s.d.

high consensus scores (Supplementary Table S3, Supplementary Figure S5b). To validate the direct association of RAR family proteins with miR-200c, we performed ChIP analysis in MCF12A cells targeting the RAR-RE using antibodies specifically against
RARa, RAR $\beta$ and RARy. We found that among these RAR family members, RAR $\beta$ was most strongly associated with the MiR-200c promoter (Supplementary Figure S5c). Specifically, ATRA induced a significant enhancement of RAR $\beta$ association to the MiR-200C 
promoter region 8 ( $r 8)$, which encompassed a putative RAR-RE (s8) right next to a $\mathrm{CpG}$ island (Supplementary Figures S5b and c). Interestingly, TET2 also showed a significant association with r8 upon ATRA treatment (Supplementary Figure S5d).
ATRA treatment consistently resulted in transcriptional activation of the luciferase driven by MiR-200c promoter, which was reversed by mutations of the RAR-RE s8 (Supplementary Figures $S 6 a$ and $b)$. The sequential-ChIP results further revealed that RAR $\beta$

\section{a High tumor grade}
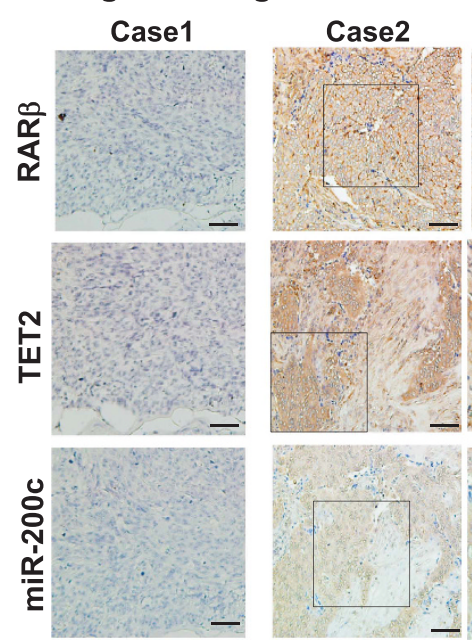

Case2 (5x)
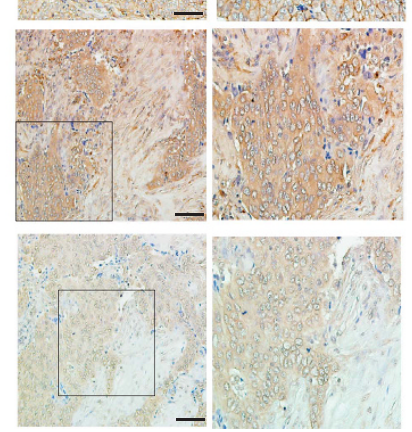

C

\begin{tabular}{|c|c|c|c|}
\hline & HG & LG & \\
\hline N-TET2+ & $11(16 \%)$ & $45(64 \%)$ & \multirow{3}{*}{$P<0.01$} \\
\hline C-TET2+ & $28(41 \%)$ & $15(21 \%)$ & \\
\hline TET2- & $30(43 \%)$ & $11(15 \%)$ & \\
\hline N-RARß+ & $11(16 \%)$ & $40(56 \%)$ & \multirow{3}{*}{$P<0.01$} \\
\hline C-RAR $\beta+$ & $13(19 \%)$ & $6(9 \%)$ & \\
\hline RAR $\beta-$ & $45(65 \%)$ & $25(35 \%)$ & \\
\hline miR200c+ & $17(25 \%)$ & $42(59 \%)$ & \multirow{3}{*}{$P<0.01$} \\
\hline miR200c- & $52(75 \%)$ & $29(41 \%)$ & \\
\hline Total & 69 & 71 & \\
\hline
\end{tabular}

e

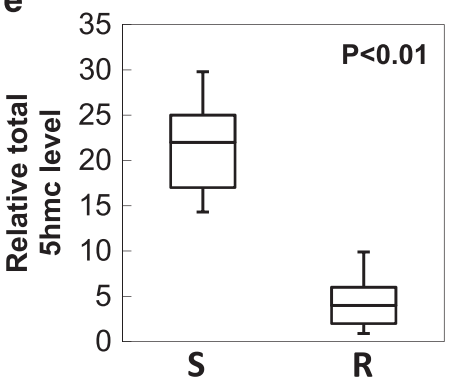

b Low tumor grade

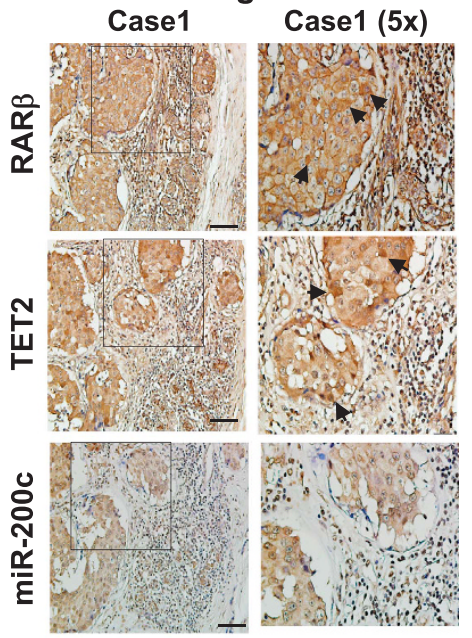

d

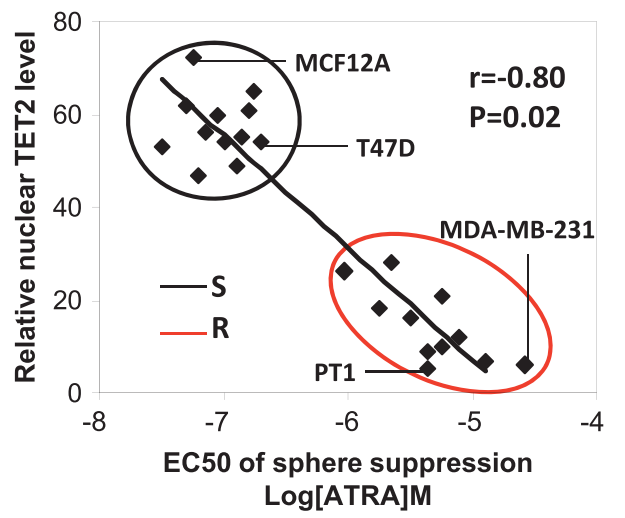

f

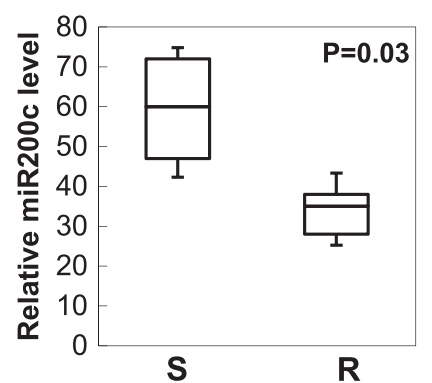

Figure 3. Lost nuclear RAR $\beta / T E T 2$ and deficient miR-200c expression is correlated with ATRA resistance in high-grade, aggressive breast cancer. (a, b) Representative IHC staining images showing expression levels of RAR $\beta$, TET2 and miR-200c in 140 human breast tissue specimens, including low-grade tumors (a: tumor grade I, LG) and high-grade tumors (b: tumor grades II-III, HG, arrowhead indicates positive nuclear staining, scale bar: $100 \mu \mathrm{m}$ ). (c) The nuclear/cytoplasmic RAR $\beta$, nuclear/cytoplasmic TET2 and total miR-200c levels were scored and their correlation with tumor grade was analyzed by Chi-square analysis. N, nuclear staining; C, cytoplasmic staining; (-), negative-low staining; (+), strong-positive staining. (d) Pearson's correlation analysis of ATRA sensitivity (S) and resistance (R) defined by ATRA-EC 50 for tumor sphere suppression with the relative expression level of nuclear TET2 showing that ATRA-EC50 is negatively correlated with nuclear TET2 level $(r=-0.8, P=0.02)$. ATRA-resistant cells $(n=11$, grouped in red circle) that include seven triple-negative primary tumor samples (for example, PT1), as well as BT549, MDA-MB-231, BT20, and SUM149PT breast cancer cell lines; ATRA-sensitive cells ( $n=12$, grouped in black circle) that include six luminal and one HER2+ primary tumor samples, as well as MCF7, T47D, ZR75-1 breast cancer cell lines and MCF10A and MCF12A cell lines. Box plots showing (e) the relative $5 \mathrm{hmc}$ levels, and (f) the relative miR-200c levels in ATRA-sensitive (S) cells and ATRA-resistant (R) cells shown in d. $R$, resistant cells with ATRA-EC $C_{50}$ of sphere suppression $\geqslant 10^{-6} \mathrm{M}$; $\mathrm{S}$, sensitive cells with ATRA-EC ${ }_{50}$ of sphere suppression $<10^{-6} \mathrm{M}$. Relative expression levels of nuclear TET2, $5 \mathrm{hmc}$, and miR-200c levels were determined by expression ratios to normalized total input lysates, total input genomic DNA and RNU6B expression, respectively. Error bars denote \pm s.d. 
along with TET2 were indeed bound to the MiR-200c promoter (Supplementary Figure S6c). ATRA significantly increased the association of both RAR $\beta$ and TET2 with the MiR-200c promoter, where the $5 \mathrm{hmc}$ level was enhanced, whereas the 5methylcytosine level was reduced (Supplementary Figures S6d and e). However, knocking-down RAR $\beta$ abolished the association between TET2 and the MiR-200c promoter with a markedly reduced $5 \mathrm{hmc}$ level (Supplementary Figures S6d and e). Together, these data suggest that RAR $\beta$ is required for recruitment of TET2 in a complex bound to miR-200c promoter region.

Lost nuclear TET2 and deficient miR-200c expression is correlated with ATRA resistance in high tumor grade and aggressive breast cancer

To further strengthen the pathological correlation of RAR $\beta$-TET2miR-200c regulation in human breast cancer, we performed a a

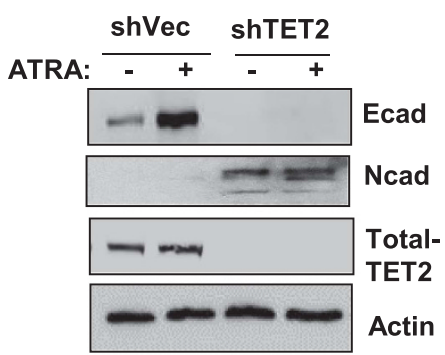

C

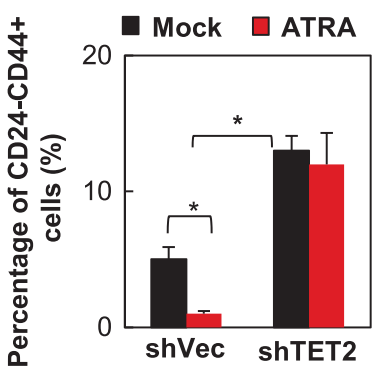

d
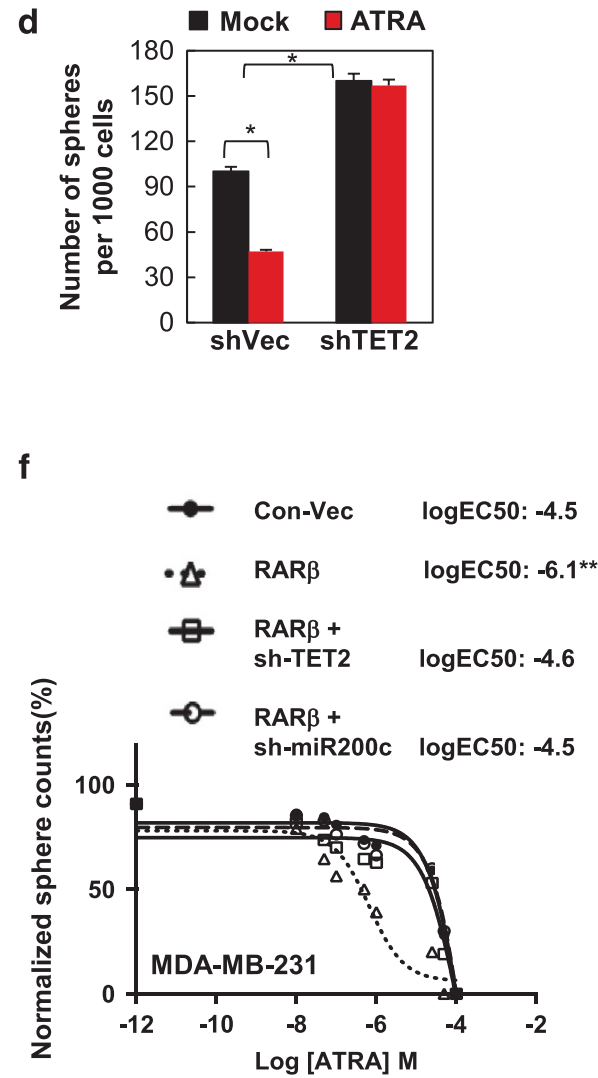

b

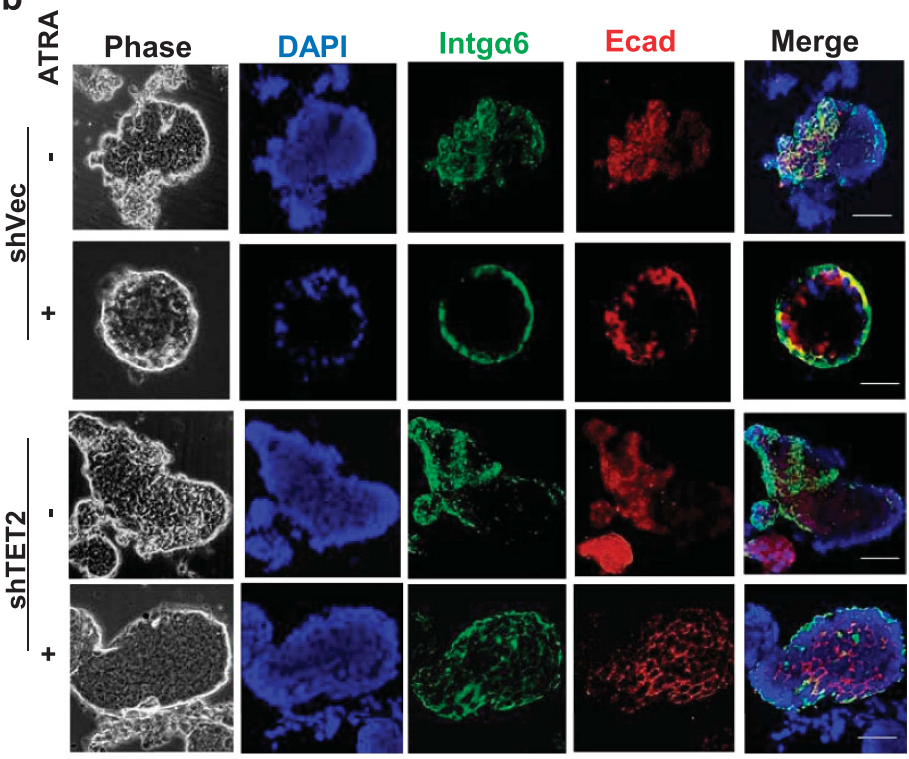

e

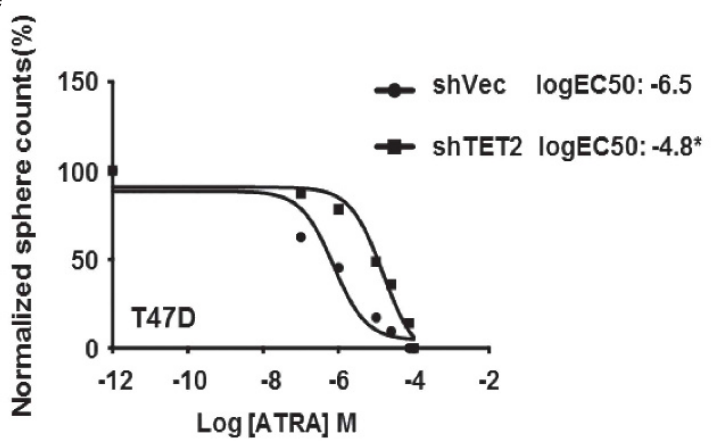

g

ATRA: - ++++

RAR $\beta:$ - + - + - +

shTET2: - - - - + +

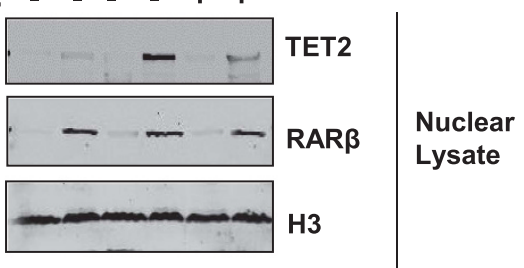

h

ATRA: - - + +

RARB: - + + +

ShTET2: - - - +

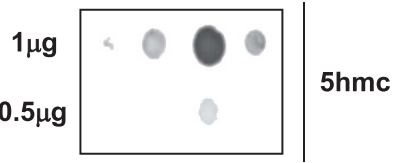


correlation analysis of RAR $\beta$ (nuclear vs cytoplasmic), TET2 (nuclear vs cytoplasmic) and miR-200c expression levels in human breast tissue microarrays consisting of a cohort of breast tumor samples. We found that RAR $\beta$ and TET2 were predominantly expressed in the nucleus of the well-differentiated low tumor grade breast tumors (LG, grade I), where miR-200c was highly expressed (Figures $3 a-c$, arrowheads indicate positive nuclear staining, $n=140, P<0.01)$. In contrast, the poorly differentiated high tumor grade tumors (HG, grades II-III) exhibited lost expression of total/ nuclear RAR $\beta$ and TET2, along with significantly reduced miR-200c levels (Figures 3a-c, $n=140, P<0.01$ ). We next examined the correlation of nuclear TET2 expression level with the CSC sensitivity to ATRA, indicated by the number of tumor spheres under treatment of various concentrations of ATRA (sensitive-S vS resistant- $R$ defined by $E_{50}$ of sphere suppression under ATRA treatment with a dose range between $10^{-8} \mathrm{M}$ and $10^{-4} \mathrm{M}$ ), in a cohort of primary human breast cancer cells (six luminal breast cancer, seven TNBC and one Her2-positive breast cancer Her2+), and non-tumorigenic immortalized mammary epithelial cell lines (MCF12A and MCF10A). Notably, TNBC, a breast cancer subtype deficient in expression of estrogen receptor (ER), progesterone receptor (PR) and HER2, tends to be more aggressive than the other subtypes and manifests high recurrence along with poor survival. ${ }^{3}$ We found that the nuclear TET2 level and the sensitivity of the spheres in response to ATRA are comparable between the non-tumorigenic mammary epithelial cell lines and the luminal/ Her2+ breast cancer cells, where nuclear TET2 was highly expressed, and ATRA-EC 50 of sphere suppression was $<10^{-6} \mathrm{M}$ (Figure 3d). However, compared with luminal/Her2+ breast cancer cells, nuclear TET2 expression was uniformly and markedly diminished in all of the primary TNBC cells, as well as in the TNBC-like breast cancer cell lines, where a resistance to ATRAmediated tumor sphere suppression was manifested (Figure $3 \mathrm{~d}$, $n=23, r=-0.8, P=0.02$ ). Consistently, $5 \mathrm{hmc}$ and miR-200c levels were significantly reduced in the ATRA-resistant cancer cells compared with the ATRA-sensitive cancer cells (Figures $3 e$ and $f$, $n=23, P<0.05)$. Together, these data indicate that deficient RARB-TET2-miR-200c regulation is functionally linked to ATRA resistance and is correlated with a poorly differentiated, TNBC phenotype.

TET2 expression is required for sensitizing breast CSCs to ATRA

Next, we attempted to determine whether ATRA-induced TET2 signaling is involved in regulation of stemness properties, including the EMT phenotype that is known to link to generation of the CSC traits. ${ }^{20}$ We found that ATRA treatment in T47D cells led to an increase in the expression of the epithelial marker, E-cadherin, which was abolished upon further knock-down of
TET2 (Figure 4a). Knockdown of TET2 also substantially increased the expression of the mesenchymal marker, N-cadherin (Figure 4a).

We further attempted to understand the role of ATRA-TET2 regulation on mammary cell stemness vs differentiation using a three-dimensional acini culture in matrigel. ${ }^{21}$ We found that T47D cells exhibited disorganized acinar formation (enlarged acini size with luminal filling), along with disrupted expression of a6integrin (basal polarity marker) and E-cadherin (epithelia marker) (Figure $4 \mathrm{~b}$ and Supplementary Figure S7), where ATRA treatment led to retrieval of the normal epithelial-like, well-differentiated acinar structure with polarized expression of a6-integrin and E-cadherin (Figure $4 \mathrm{~b}$ and Supplementary Figure S7). ATRA treatment consistently resulted in a substantial reduction in the putative stem cell population marked by $\mathrm{CD} 24^{-} \mathrm{CD} 44^{+}$or enriched by the side population using Hoechst dye (Figure 4c, Supplementary Figures S8a and b), and also suppressed the number of spheres, suggesting a diminished stem cell pool under ATRA treatment (Figure 4d: no significant changes in cell death or cell cycle profile; data not shown). In contrast, knockdown of TET2 enhanced the $\mathrm{CD} 24^{-} \mathrm{CD} 44^{+}$population and the side population (Figure 4c, Supplementary Figures S8a and b), along with increasing the sphere formation and abolishing ATRAmediated effects on cell polarization and acinar formation (Figures $4 \mathrm{~b}$ and $\mathrm{d}$ ).

We also found that T47D cells were sensitive to ATRA, which blocked $50 \%$ of the sphere formation with a half maximal effective concentration $\left(E_{50}\right.$ ) at $10^{-6.5} \mathrm{M}$ (Figure $4 \mathrm{e}$ ). However, knockdown of TET2 significantly diminished the sensitivity of T47D cells to ATRA as indicated by the elevated $\mathrm{EC}_{50}$ (to $\mathrm{EC}_{50}=10^{-4.8} \mathrm{M}$, Figure 4e). In contrast to T47D, sphere formation in MDA-MB-231 cells, which exhibited a deficiency in endogenous RAR $\beta$ expression and TET2 nuclear localization as shown in Figure 1g, was highly resistant to ATRA treatment $\left(E C_{50}=10^{-4.5} \mathrm{M}\right.$, Figure $\left.4 \mathrm{f}\right)$. We found that ectopic expression of RAR $\beta$ in MDA-MB-231 cells was able to sensitize the cells to ATRA treatment (to $\mathrm{EC}_{50}=10^{-6.1} \mathrm{M}$, Figure $4 \mathrm{f}$ ), accompanied by the enhanced nuclear TET2 expression and increased $5 \mathrm{hmc}$ level (Figures $4 \mathrm{~g}$ and $\mathrm{h}$ ), whereas further knockdown of TET2 diminished $5 \mathrm{hmc}$ expression and reversed ATRA sensitization caused by RAR $\beta$ re-expression (Figures $4 \mathrm{f}-\mathrm{h}$ ).

Our data in Figure 2 suggest that miR-200c is a major target of ATRA-TET2 in regulation of stemness. Indeed, knockdown of miR-200c in ATRA-sensitive T47D cells promoted sphere formation and tumor-seeding capacity (Supplementary Figures S8c and d), which were previously inhibited upon ATRA treatment. However, ectopic expression of miR-200c sensitized ATRA-resistant cells MDA-MB-231 to ATRA as evidenced by significantly reduced sphere formation and abolished xenograft tumor formation,

Figure 4. TET2 activation is required for breast CSC sensitivity to ATRA. (a) Immunoblots showing protein expression levels of E-cadherin (epithelial marker) and N-cadherin (mesenchymal marker) in T47D breast cancer cells that stably expressed sh-TET2 or sh-Vec and treated with $10^{-6} \mathrm{M}$ ATRA or vehicle for 3 days. (b) T47D-sh-TET2 and T47D-sh-Vec single-cell suspensions were subjected to three-dimensional (3D) ontop matrigel culture (20000 cells per well in 24-well plate) under $10^{-6} \mathrm{M}$ ATRA or vehicle treatment for 12 days (treatment medium was replenished every 2 days); acini were stained for basal polarity marker ( $\alpha 6$-integrin, green), epithelial marker (E-cadherin, red) and DAPI (nucleus, blue), and then subjected to confocal immnunofluorscence microscopic analysis (scale bar: $50 \mu \mathrm{m}, 600$ acini were imaged and analyzed from three independent experiments, also see Supplementary Figure S7). (c) The percentage of the CD24 ${ }^{-} \mathrm{CD} 44^{+}$population isolated from T47D-sh-TET2 or T47D-sh-Vec cells treated with $10^{-6} \mathrm{M}$ ATRA or vehicle for 3 days $(n=3$ independent experiments, asterisk indicates $P<0.05)$. (d) The number of spheres (sphere size $>100 \mu \mathrm{m}$ ) per 1000 initially plated T47D-sh-TET2 or T47D-sh-Vec cells treated with $10^{-6} \mathrm{M}$ ATRA or vehicle for 7 days. ( $n=3$ independent experiments, asterisk indicates $P<0.05$ ). (e) The dose response was plotted and EC50 was calculated using GraphPad Prism software (La Jolla, CA, USA), based on the number of the spheres generate from T47D-sh-TET2 or T47D-shVec cells treated with ATRA or vehicle for 7 days with the dose range between $10^{-12} \mathrm{M}$ and $10^{-4} \mathrm{M}$ (where $Y$ axis is the normalized sphere number counts (\%) and $X$ axis is the logarithm of (ATRA) concentration, $n=3$ independent experiments, asterisk indicates $P<0.05$ ). (f) Doseresponse curve (normalized sphere number (\%) vs log (ATRA) M), (g) nuclear TET2 and RAR 32 protein expression levels and (h) total 5hmc levels shown by $5 \mathrm{hmc}$ dot blot using diluted genomic DNA ( 1 and $0.5 \mu \mathrm{g}$ ) of MDA-MB-231 breast cancer cells that expressed RAR $\beta$, RAR $\beta+$ shTET2, RAR $\beta+$ sh-miR-200c or sh-Vec under ATRA or vehicle treatment ( $n=3$ independent experiments, double asterisk indicates $P<0.01)$. Comparable amount of control vector and vehicle were included as negative control (-) in each group. Error bars denote \pm s.d. 
suggesting a markedly diminished CSC pool (Supplementary Figures $\mathrm{S} 8 \mathrm{e}$ and $\mathrm{f}$ ). Together, these data reveal the critical role that TET2-miR-200c has in regulation of breast CSC sensitivity to ATRA treatment.

ATRA downregulates PKC $\zeta$ through activation of miR-200c We next attempted to identify novel gene targets and the associated mechanism regulated by TET2-miR-200c in response to ATRA. Using global gene expression profiling analysis of T47D cells that were under ATRA treatment compared with that of the mock- treated cells, combined with the analysis result of miR-200c seed sequence matching targets from three miRNA-target prediction databases (DIANSmT, miRand and TargetScan), 26 putative miR-200c targets were identified with expression levels that were significantly downregulated by ATRA and also with high context and conservation seed sequence matching scores of miR-200c interaction (Figure 5a, TargetScan 6.0); among these candidates,

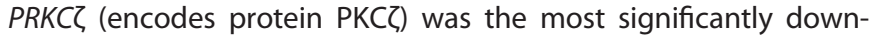
regulated gene under ATRA treatment (Figure 5a, 9.7-fold change, $n=3$, false discovery rate $<0.05, P<0.01)$. PKC $\zeta$, a member of the aPKC family, has an evolutionarily conserved role in directing self- a

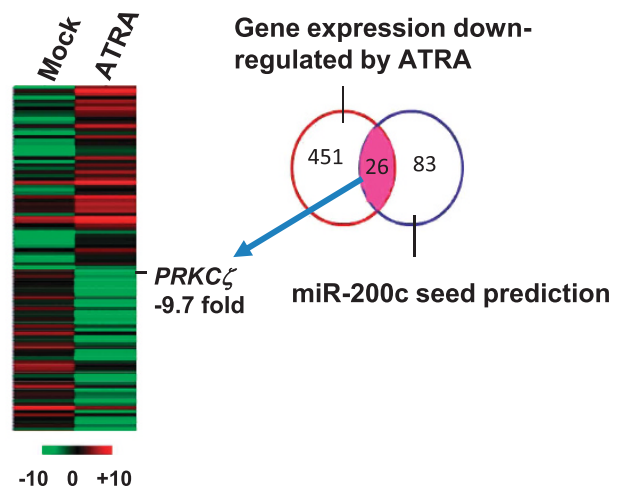

d

\begin{tabular}{|c|c|}
\hline 3' AGGUAGUAAUGGGCCGUCAUAAU $5^{\prime}$ & hsa-miR-200c \\
\hline|||||| & \\
\hline 5' GTCAUGUUUUAAAAGGAGUUUUU & PRKC $\zeta$ \\
\hline $\begin{array}{l}\text { 3' AGGUAGUAAUGGGCCG-UCAUAAU 5' }^{\prime} \\
||||||||||\end{array}$ & $m m$ \\
\hline 5' GUUGUUAAUAUUUAACAAGUAUUU 3' & Prkc $\zeta$ \\
\hline
\end{tabular}

f

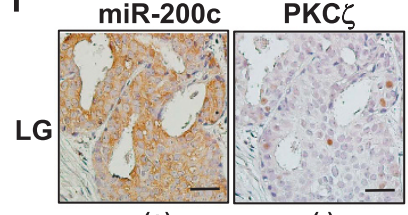

$(+)$

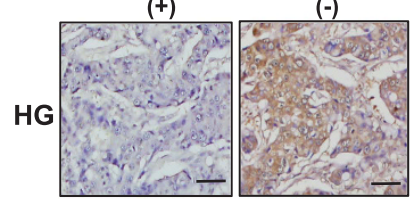

$(-)$

$(+)$ b

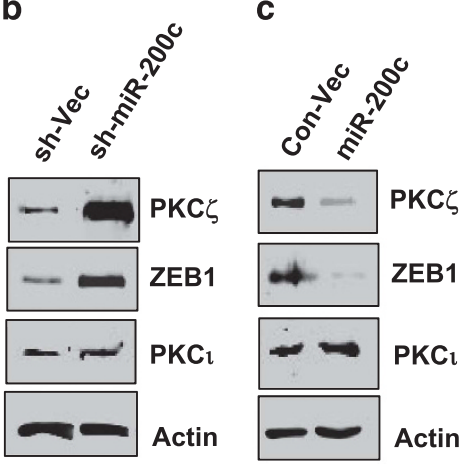

e

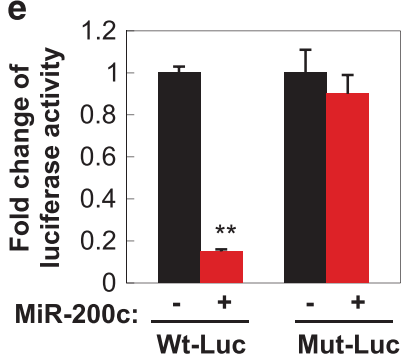

\begin{tabular}{|c|c|c|}
\hline $\begin{array}{l}\text { Tumor } \\
\text { Grade }\end{array}$ & LG & HG \\
\hline miR200c+ & $15(15 \%)$ & $10(10 \%)$ \\
\hline miR200c- & $11(12 \%)$ & $62(63 \%)$ \\
\hline Total & $26(27 \%)$ & $72(73 \%)$ \\
\hline PKC $\zeta+$ & $7(7 \%)$ & $41(42 \%)$ \\
\hline PKC $\zeta=$ & $19(19 \%)$ & $31(32 \%)$ \\
\hline Total & $26(26 \%)$ & $72(74 \%)$ \\
\hline
\end{tabular}

h

1: Non-CD44+CD24

2: CD44+CD24:

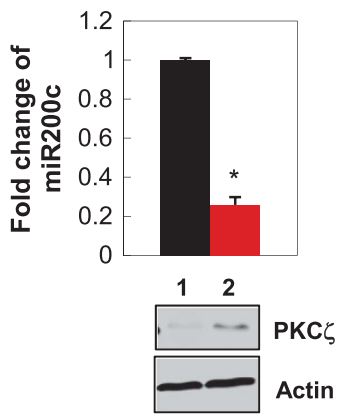

i Con-Vec sh-TET2 miR200c ATRA:

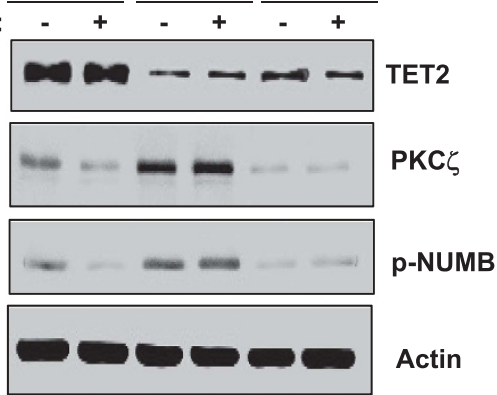

j

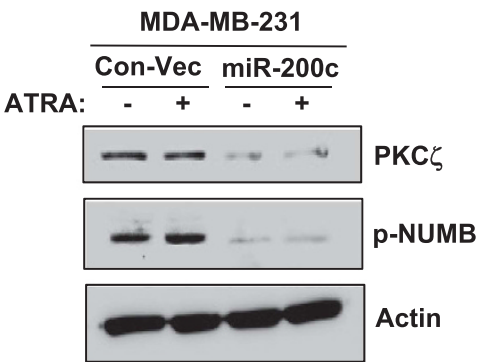


renewing $A D$ of mammalian stem cells. ${ }^{5-7}$ Notably, PKC expression is linked to breast cancer invasiveness and metastasis. $^{22}$

Based on the data shown in Figure 5a, we speculated that PKC乙 could be regulated by ATRA as a new miR-200c target. Immunoblotting data first validated that PKC was markedly upregulated by knockdown of miR-200c, while it was downregulated by ectopic expression of miR-200c in T47D cells (Figures $5 \mathrm{~b}$ and $\mathrm{c}$ ). To further determine whether PKC $\mathrm{P}$ is directly regulated by miR-200c through interaction of the complementary $3^{\prime}$-UTR region (conserved between human and mouse Figure 5d), a luciferase reporter linked with 3'-UTR of PKCZ was used. Indeed, expression of miR-200c significantly decreased the luciferase activity driven by PKC -UTR, whereas a reporter mutated in PKC $-3^{\prime}$-UTR failed to respond to miR-200c expression (Figure 5e).

Next, to determine whether miR-200c-PKCद regulation is functionally linked to the cancer phenotype and to the stem cell traits, we first examined PKC and miR-200c expression levels in human breast tissue sections consisting of a cohort of normal and malignant breast tissue samples. We found that miR-200c was most abundantly expressed in benign lesions and welldifferentiated low-grade (LG, tumor grade $\leqslant$ I) breast tumors and was negatively correlated with PKC expression (Figures $5 e$ and f). In contrast, PKC 3 was overexpressed in high-grade $(\mathrm{HG}$, tumor grades II-III) aggressive breast tumors, where miR-200c was most repressed (Figures $5 f$ and $g, n=98, P<0.01$ ). We then examined PKC $\zeta$ and miR-200c expression levels in the CSC-enriched CD24 $\mathrm{CD} 44^{+}$cell population compared with the non-CD24 ${ }^{-} \mathrm{CD} 44^{+}$cell population isolated from primary human breast tumor cells. Compared with the non-CD24-CD44 ${ }^{+}$cell population, PKCद protein was found to be highly expressed in the $\mathrm{CD} 24^{-} \mathrm{CD} 44^{+}$ cell population, where miR-200c was repressed (Figure $5 \mathrm{~h}$ ).

Furthermore, we found that ATRA treatment significantly decreased PKCZ expression in ATRA-sensitive T47D cells, whereas knockdown of TET2, which was shown to suppress the miR-200c level in Figure 2, markedly increased PKC expression. The

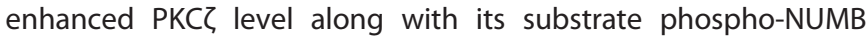
( $p-N U N B$ ) was diminished upon re-expression of miR-200c in T47D cells (Figure 5i), as well as in ATRA-resistant MDA-MB-231 cells (Figure 5j).

ATRA directs breast cancer cell state changes and decreases the CSC pool through miR-200c-mediated suppression of PKCZ

Our data in Figure 5 showed that PKCZ, a critical member of the aPKC family, was a putative target of miR-200c under the regulation of ATRA-TET2 signaling. Therefore, we next examined whether TET2-miR-200c-PKC signaling could have a role in regulation of the breast cancer cell state in response to ATRA.

We have previously established a paired cell imaging analysis to examine the polarity of breast CSC division by analyzing the intracellular distribution of the cell fate determinant NUMB along with the breast CSC surface marker CD44 immediately after mitosis by treatment with blebbistatin, a small molecule that arrests cytokinesis to form a binucleated cell. ${ }^{19}$ Using paired cell analysis of the isolated $\mathrm{CD} 24^{-} \mathrm{CD} 44^{\text {high }}$-CSC population from T47D cells, the data revealed that in the dividing CSC that exhibited the $A D$ pattern, $P K C \zeta$ was localized to the progeny cell expressing the putative stem cell surface marker CD44 (Figures $6 a$ and b, Supplementary Figure S9), whereas NUMB was exclusively polarized to the CD44-negative progeny cell (Figures $6 a$ and b). Moreover, in the dividing CSC that exhibited the SC pattern, NUMB was highly expressed and uniformly distributed in the binucleated cell, where CD44 was lost in both progenies; in the dividing CSC that exhibited the SD pattern, NUMB was repressed while CD44 was highly expressed and uniformly distributed in both progenies of the binucleated cell (Figures $6 a$ and $b$ ).

Our data here also showed that ATRA treatment in the ATRAsensitive T47D cells significantly diminished PKCZ and p-NUMB levels and led to a substantial increase in the frequency of the SC pattern, along with a decrease in the $A D$ pattern of the CSC population (there was no significant change in the SD pattern); however, the ATRA-mediated enhancement of SC pattern was abolished upon ectopic expression of PKC (Figures $6 c$ and d). In contrast, knockdown of TET2 cells in T47D cells (T47D-sh-TET2) resulted in resistance to ATRA, whereas treatment of the PKCZ inhibitor along with ATRA led to a markedly increased frequency of the SC pattern along with a reduced frequency of the $A D$ pattern (Figures $6 \mathrm{e}$ and $\mathrm{f}$ ). Similar results were able to be recapitulated in the ATRA-resistant MD-MB-231 cells where

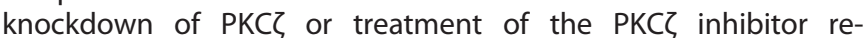
sensitized the CSCs to ATRA-enhanced SC pattern (Figures $6 \mathrm{~g}$ and h), whereas re-expression of PKCZ reversed the effects caused by knock-down of PKCZ (Supplementary Figure S10).

To determine the role of the TET2-miR-200c-PKCZ signaling axis in regulating the CSC pool, the isolated $\mathrm{CD} 24^{-} \mathrm{CD} 44^{\text {high }}-\mathrm{CSC}$ population from T47D or MDA-MB-231 cells was subjected to a serial sphere formation assay. ATRA treatment in T47D cells substantially diminished sphere formation at each passage (Figure 6i), suggesting that an decreased CSC population; however, the ATRA-diminished CSC pool was able to be fully rescued upon re-expression of PKC (Figure 6i). Furthermore, ATRA treatment did not affect sphere formation in both T47D-sh-TET2 cells and MD-MB-231 cells, where the primary spheres were around the same number of the secondary and tertiary spheres

Figure 5. ATRA treatment leads to downregulation of $\mathrm{PKC} \zeta$, a new miR-200c target. (a) Heat map showing mean expression values of the putative miR-200c target genes with expression levels that were most significantly downregulated in $10^{-6} \mathrm{M}$ ATRA-treated T47D cells for $24 \mathrm{~h}$ ( $n=3$ independent experiments, fold change $>2$ compared with vehicle-treated cells, $P<0.05$ ). Putative miR-200c targets were identified by seed sequence matching from three prediction databases (DIANSmT, miRand and TargetScan). (b) Immunoblots showing PKC $\zeta$ expression levels in T47D cells that stably expressed sh-miR-200c and the control vector. (c) Immunoblots showing PKC $\zeta$ expression levels in T47D cells

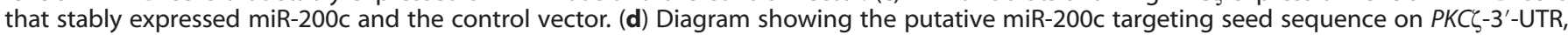
which was conserved between human and mouse (underline indicating mutation of UUU to CCC in the mutated PKC - $3^{\prime} U T R$ luciferase reporter, Mut-Luc). (e) Fold change of luciferase activity driven by the wild-type or mutant $P K C \zeta-3^{\prime}-U T R$ reporter in T47D cells that stably expressed miR-200c and treated with $10^{-6} \mathrm{M}$ ATRA or vehicle for $24 \mathrm{~h}(n=3$ independent experiments, double asterisk indicates $P<0.01)$. (f) Representative IHC staining images showing a reverse correlation between expression of miR-200c and PKC $\zeta$ in human breast tumor sections. (-), negative-low staining; $(+)$, strong-positive staining. (g) Chi-square correlation analysis of the expression levels of PKC $\zeta$ and miR-200c with the tumor grade ( $n=98, P<0.01$; tumor grades II-III, HG; tumor grade I, LG). (h) miR-200c and PKC $\zeta$ expression levels in the CD $44^{+} \mathrm{CD} 24^{-}$population versus the non-CD44 ${ }^{+} \mathrm{CD} 24^{-}$population isolated from primary human breast tumor cells $(n=3$ independent experiments, asterisk indicates $P<0.05$ ). (i) Protein expression in T47D cells that stably expressed miR-200c, sh-TET2, or the control vectors and treated with $10^{-6} \mathrm{M}$ ATRA or vehicle for $24 \mathrm{~h}$. (j) Protein expression in MDA-MB-231 cells that stably expressed miR-200c or the control vector and treated with $10^{-6} \mathrm{M}$ ATRA or vehicle for $24 \mathrm{~h}$. Comparable amount of control vector and vehicle were included as negative control $(-)$ in each group. Error bars denote \pm s.d. 
a

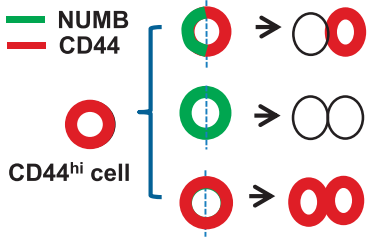

Asymmetric

Division (AD)

Symmetric

Commitment (SC)

Symmetric

Division (SD)

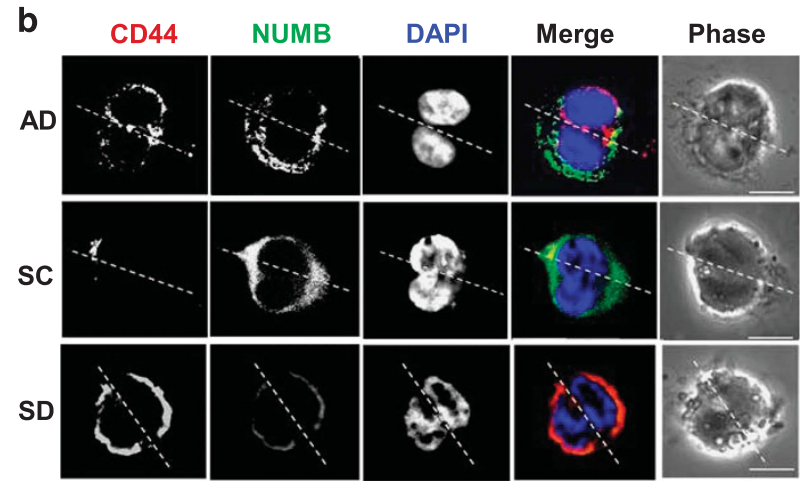

C

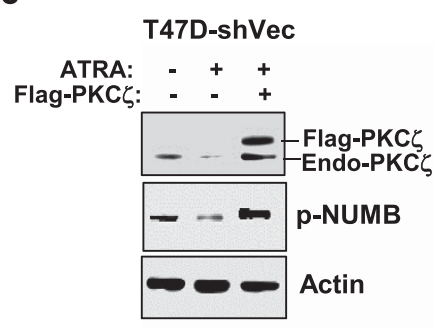

e

T47D-shTET2

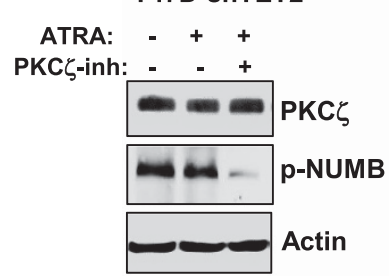

g

MDA-MB-231

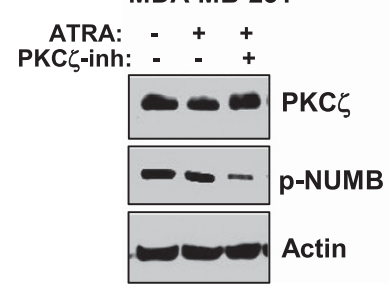

d

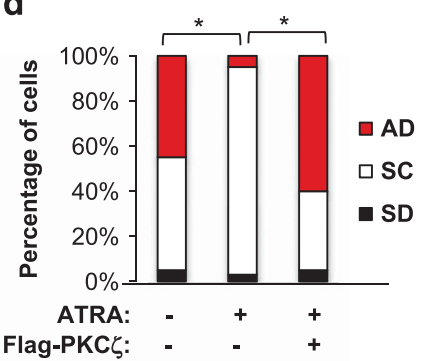

f

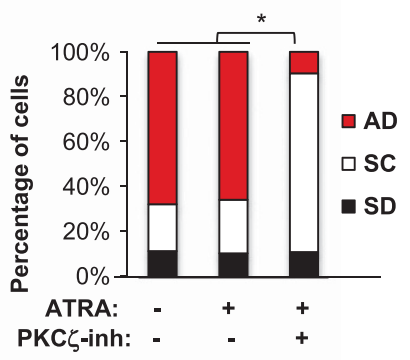

h

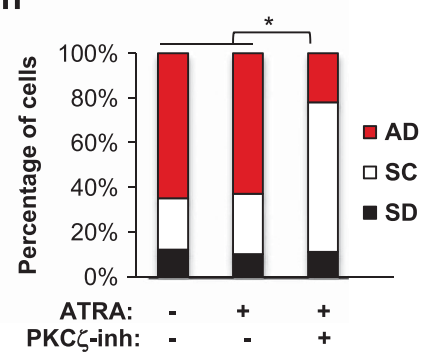

I Primary sphere

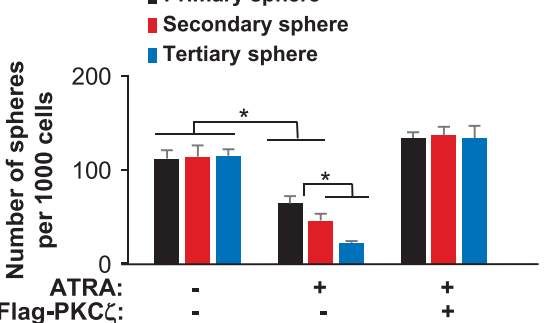

Flag-PKC $\zeta$ :

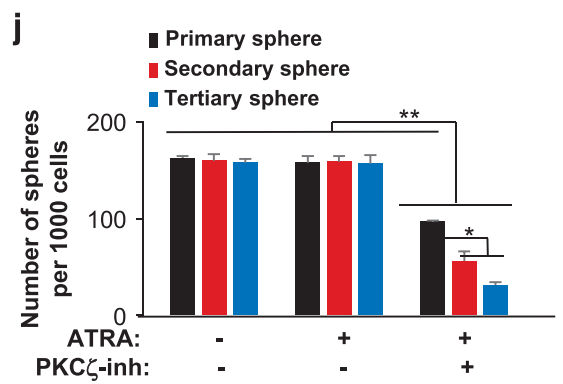

k

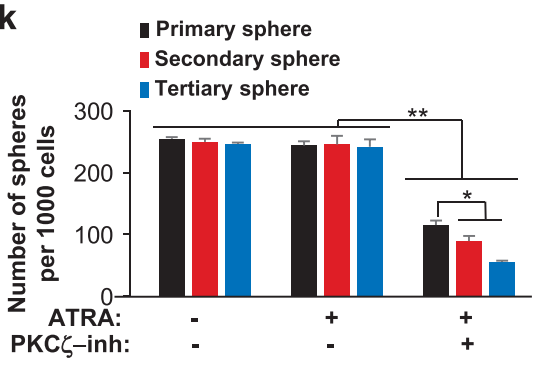

Figure 6. ATRA directs breast cancer cell state changes and decreases breast CSCs through suppression of PKC division patterns: AD, SD or SC patterns. (b) Representative confocal fluorescence images showing intracellular distribution of CD44 (red) and NUMB (green), in the dividing CD24-CD44 ${ }^{\text {hi }}$ cells isolated from T47D cells using paired cell analysis (scale bar: $10 \mu \mathrm{m}$ ). (c) Protein expression levels of PKC $\zeta$ and p-NUMB in T47D cells that ectopically expressed PKC $\zeta$ or the control vector and treated with $10^{-6} M$ ATRA or vehicle for $24 \mathrm{~h}$ and (d) the percentages of these cells that manifested AD, SC and SD division patterns $(n=700-1000$ randomly selected cells from three independent experiments, asterisk indicates $P<0.05)$. (e) Protein expression levels of PKC $\zeta$ and p-NUMB in T47D-sh-TET2 cells that were treated with PKC $\zeta$ pseudo substrate inhibitor $(25 \mu \mathrm{M})$, ATRA $\left(10^{-6} \mathrm{M}\right)$, ATRA with PKC $\zeta$ inhibitor or vehicle for $24 \mathrm{~h}$, and (f) the percentages of these cells that manifested AD, SC and SD division patterns $(n=700-1000$ randomly selected cells from three independent experiments, asterisk indicates $P<0.05)$. (g) Protein expression levels of PKC $\zeta$ and $\mathrm{p}-\mathrm{NUMB}$ in MDA-MB-231 cells that were treated with PKC $\zeta$ pseudo substrate inhibitor $(25 \mu \mathrm{M})$, ATRA $\left(10^{-6} \mathrm{M}\right)$, ATRA with PKC $\zeta$ inhibitor or vehicle for $24 \mathrm{~h}$, and (h) the percentages of these cells that manifested AD, SC and SD division patterns ( $n=700-1000$ randomly selected cells from three independent experiments, asterisk indicates $P<0.05)$. (i) The number of the serially passaged tumor spheres (sphere size $>100 \mu \mathrm{m}$ ) per 1000 initially plated T47D cells that ectopically expressed PKC $\zeta$ or the control vector and treated with $10^{-6} \mathrm{M}$ ATRA or vehicle for $24 \mathrm{~h}$ ( $n=3$ independent experiments, asterisk indicates $\left.P<0.05\right)$. (j) The number of the serially passaged tumor spheres (sphere size $>100 \mu \mathrm{m}$ ) per 1000 initially plated T47D-sh-TET2 cells that were treated with PKC $\zeta$ pseudo substrate inhibitor $(25 \mu \mathrm{M})$, ATRA $\left(10^{-6} \mathrm{M}\right)$, ATRA with PKC $\zeta$ inhibitor or vehicle for $24 \mathrm{~h}(n=3$ independent experiments, asterisk indicates $P<0.05$, double asterisk indicates $P<0.01)$. (k) The number of the serially passaged tumor spheres (sphere size $>100 \mu \mathrm{m})$ per 1000 initially plated MDA-MB-231 cells that were treated with PKC $\zeta$ pseudo substrate inhibitor $(25 \mu \mathrm{M})$, ATRA (10 $\left.{ }^{-6} \mathrm{M}\right)$, ATRA with PKC $\zeta$ inhibitor, or vehicle for $24 \mathrm{~h}$ ( $n=3$ independent experiments, asterisk indicates $P<0.05$, double asterisk indicates $P<0.01)$. Comparable amount of control vector and vehicle were included as negative control $(-)$ in each group. Error bars denote \pm s.d. 
(Figures $6 \mathrm{j}$ and $\mathrm{k}$ ), suggesting that a constant CSC population was maintained. But treatment of the PKC inhibitor or knockdown of PKC $\zeta$ led to a continuing diminishment of the spheres at each passage (Figures $6 \mathrm{j}$ and k, Supplementary Figure S10), whereas reexpression of $\mathrm{PKC} \zeta$ reversed the effects caused by knockdown of PKC (Supplementary Figure S10).

Consistently, downregulation of PKC by enforced expression of miR-200c in primary human breast tumor cells manifested ATRA resistance (PT1, Figure $3 \mathrm{~d}$ ) led to a decreased frequency of the AD pattern and significant suppression of the CSC pool, as evidenced by reduced $\mathrm{CD} 24^{-} \mathrm{CD} 44^{+}$population, diminished tumor sphere formation at each passage for three consecutive passages, and inhibited mammary xenograft tumor formation with a significant reduction in the tumor-seeding CSC frequency (Supplementary Figure S11), whereas re-expression of PKC reversed the effects caused by expression of miR-200c (Supplementary Figure S11).

Furthermore, using a three-dimensional acinar differentiation culture in matrigel, the data showed that ATRA-resistant MDMB-231 cells, which highly expressed the basal/myoepithelial lineage marker a-SMA, failed to form the normal acinar structure even in the presence of ATRA (Supplementary Figure S12), whereas PKC inhibitor treatment combined with ATRA was able to reinforce a well-differentiated acinar morphology with predominant expression of the luminal lineage marker CK18, pointing to a commitment to the luminal cell-like state (Supplementary Figure S12). Compared with treatment of ATRA or PKC inhibitor alone, in vivo treatment of PKC 3 inhibitor along with ATRA treatment significantly suppressed MDA-MB-231 xenograft breast tumor growth and tumor volume (Supplementary Figures S13a and $b)$, and caused the poorly differentiated high-grade adenocarcinoma phenotype to revert to a well-differentiated low-grade cancer phenotype (Supplementary Figure S13c). In addition, PKCC inhibitor effectively inhibited p-NUMB in the tumor tissues, promoted the luminal cell lineage with a robust expression of CK18 (Supplementary Figure S13c), and also abolished in vitro serial tumor sphere formation of the isolated xenograft tumor cells from the treated mice (Supplementary Figure S13d). Together, these data suggest that ATRA-TET2 has a role in regulation of the breast cancer cell state through suppression of PKC expression.

Inhibition of PKCZ suppresses the ATRA-resistant CSC pool and directs CSCs to the luminal cell-like state and re-sensitization to TAM

To further determine the role of PKC (encoded by $P R K C Z$ gene) in modulation of the breast cancer cell state and breast tumor progression, PRKCZ-knockout (PRKCZ-KO) MDA-MB-231 cells (Figure 7a) were generated by the CRISPR/Cas9 system. $^{23}$ We previously showed that inhibition of PKC directed MDA-MB-231 CSCs to the luminal cell-like state in vitro and in vivo (re-expression of luminal lineage markers CK18/MUC1, Figure 6 and Supplementary Figure S13), we then asked whether these luminal-like cells also expressed ERa/PR, a major characteristic of luminal subtype breast cancer, and became sensitized to the traditional first-line selective ER modulator treatments for breast cancer, such as tamoxifen (TAM). Indeed, we found that compared with the MDA-MB-231 control cells (TNBC, ER/PR/Her2-negative), the PRKCZ-KO MDA-MB-231 cells exhibited repressed $\mathrm{p}-\mathrm{NUMB}$ expression along with strong ERa expression (Figures $7 a$ and $b$ ). In addition, consistent with the PKC inhibitor treatment (Supplementary Figure S13), knockout of $P R K C Z$ markedly inhibited mammary xenograft tumor formation with significant decrease in the tumor-seeding CSC frequency (Figure 7c).

To further determine whether knockout of $P R K C Z$ could re-sensitize MDA-MB-231 cells to TAM treatment and block tumor progression in vivo, PRKCZ-KO MDA-MB-231 or the control cells were first injected into mammary fat pads of 6-week-old female nude mice, and when the tumors reached $100 \mathrm{~mm}^{3}$, the animals were given TAM (i.p., $5 \mathrm{mg} / \mathrm{kg}$, every other day, dosing based on Kumar et $a l_{.}{ }^{24}$ ), or vehicle for 2 weeks ( $n=5$ mice per each group). We found that TAM alone did not have significant effects on MDAMB-231 xenograft tumor growth, CSC frequency, tumor phenotype or serial tumor sphere formation of the isolated xenograft tumor cells from the treated mice (Figures 7c-g). However, knockout of $P R K C Z$ significantly suppressed tumor growth, abolished serial tumor sphere formation and caused the poorly differentiated high-grade adenocarcinoma phenotype to revert to a well-differentiated low-grade cancer phenotype with a robust expression of ERa (Figures $7 \mathrm{c}-\mathrm{g}$ ). Furthermore, we found that $P R K C Z-K O$ combined with TAM resulted in therapeutic synergism in abrogating tumor growth (Figure 7d). Together, these data suggest that inhibition of $\mathrm{PKC}$ directs luminal (ER+) differentiation and thereby re-sensitizes TNBC cells to TAM-mediated tumor suppression.

\section{DISCUSSION}

Our findings reveal that defective RAR $\beta / T E T 2$ signaling in aggressive tumors promotes an ATRA-resistant breast CSC pool, and miR-200c therapy or strategies that directly inhibit PKC likely direct the resistant breast CSCs to the differentiation state, potentially by enhancing $S C$ along with repressing $A D$ of these CSCs (Figures 6d-i), and thereby diminish the CSC pool (Figure 8). It is known that aPKC family members, including $\mathrm{PKC \zeta}$, can serve as tumor suppressors or tumor promoters among various cancer types. ${ }^{25,26}$ Several studies have implied that in breast cancer, PKCZ expression likely promotes cancer cell growth and aggressiveness. ${ }^{27-29}$ Furthermore, our data have shown that ATRA specifically downregulates $\mathrm{PKC \zeta}$, but not the highly homologous isoform PKCI (Supplementary Figure S14a). Downregulation of

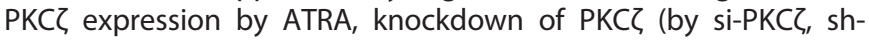

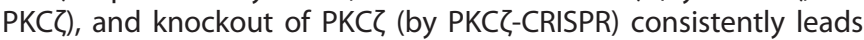
to decreased p-NUMB (Figure 7, Supplementary Figures S10 and S14), whereas knockdown of PKCI does not affect p-NUMB level (Supplementary Figure S14c). Together with the in vivo and in vivo functional data using PKC inhibitor along with $\mathrm{PKC}$-knock-down and knockout systems (Figures 6 and 7, Supplementary Figures S10 and S13), our evidence suggests that PKC is the major aPKC isoform that sustains the breast cancer CSC pool to promote breast tumor progression.

\section{MATERIALS AND METHODS}

Cell culture and treatment

The immortalized mammary epithelial cell line, MCF12A and MCF10A, and the breast cancer cell lines, including MDA-MB-231, T47D, ZR75-1, BT549 and MCF7, etc., were purchased from American Type Culture Collection (ATCC, Manassas, VA, USA). Primary breast tumor cells were purchased from Promab Inc (Richmond, CA, USA). All the cell lines were tested for mycoplasma free and authenticated using short tandem repeat profiling by ATCC within 6 months of laboratory use and cultured according to the vendor's instruction.

\section{TAM treatment in mammary xenograft tumor}

Isolation, infection and culture of tumor spheres from tumors were performed as described previously. ${ }^{30}$ In all, $10^{5}$ CRISPR-PRKCZ-knockout MDA-MB-231 cells or the control MDA-MB-231 cells were injected into mammary fat pads of 6-week-old female nude mice. When tumors reached the indicated palpable size $\left(\sim 100 \mathrm{~mm}^{3}\right)$, animals were given $100 \mu \mathrm{l}$ of corn oil (i.p. every 2 days) or TAM ( $5 \mathrm{mg} / \mathrm{kg}$ in $100 \mu \mathrm{l}$ of corn oil, i.p. every 2 days) for 2 weeks (Sigma, St Louis, MO, USA; regimen based on Kumar et al. ${ }^{24}$ and manufacturer's suggestion). For ATRA and PKC inhibitor treatment, animals were given vehicle dimethylsulfoxide or ATRA $7.5 \mathrm{mg} / \mathrm{kg}$ i.p. daily for 2 weeks (Sigma, regimen based on previous study in Elstner et al. ${ }^{31}$ )

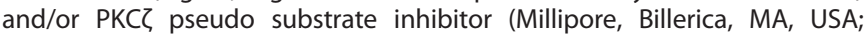
$20 \mathrm{mg} / \mathrm{kg}$ i.p. every other day for 2 weeks, regimen according to the manufacturer's suggestion). Tumor was measured by caliper every other 2 days and tumor volume was calculated using (tumor length $\times$ tumor 


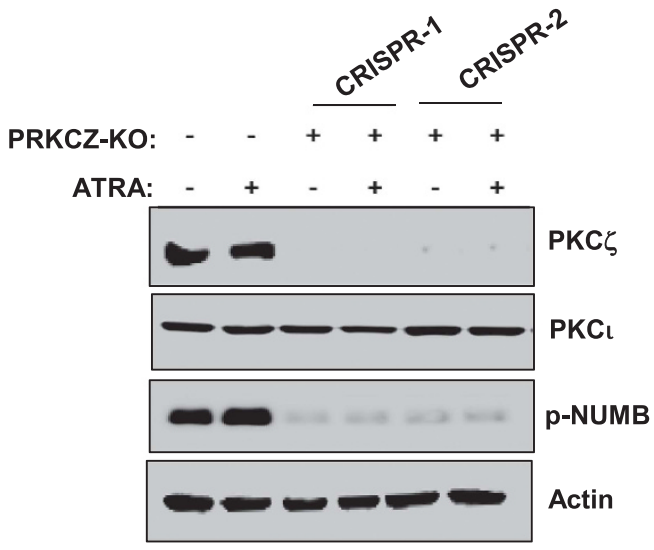

c

\begin{tabular}{|lccccc|}
\hline $\begin{array}{l}\text { Injected } \\
\text { cell \# }\end{array}$ & $1 \times 10^{5}$ & $1 \times 10^{4}$ & $1 \times 10^{3}$ & 100 & $\begin{array}{c}\text { csc } \\
\text { frequency }\end{array}$ \\
Mock & $5 / 5$ & $4 / 5$ & $4 / 5$ & $2 / 5$ & $1 / 704$ \\
TAM & $5 / 5$ & $4 / 5$ & $4 / 5$ & $3 / 5$ & $1 / 594$ \\
$\begin{array}{l}\text { PRKCZ- } \\
\text { KO }\end{array}$ & $3 / 5$ & $2 / 5$ & $0 / 5$ & $0 / 5$ & $1 / 26,892^{* *}$ \\
$\begin{array}{l}\text { TAM+ } \\
\text { PRKCZ- }\end{array}$ & & & & & \\
KO & $2 / 5$ & $2 / 5$ & $1 / 5$ & $0 / 5$ & $1 / 23,578^{* *}$ \\
\hline
\end{tabular}

e

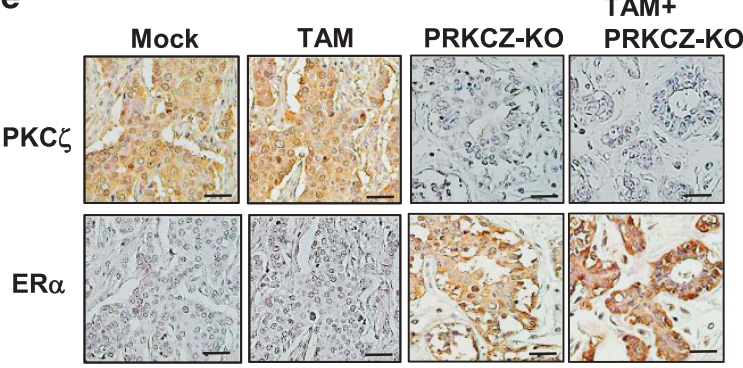

f

\begin{tabular}{|c|c|c|c|c|}
\hline & Mock & TAM & $\begin{array}{c}\text { PRKCZ } \\
-K O\end{array}$ & $\begin{array}{c}\text { TAM+ } \\
\text { PRKCZ } \\
\text {-KO }\end{array}$ \\
\hline PKC $\zeta+$ & $\begin{array}{c}5 / 5 \\
(100 \%)\end{array}$ & $\begin{array}{c}5 / 5 \\
(100 \%)\end{array}$ & $\begin{array}{c}0 / 5 \\
(0 \%)\end{array}$ & $\begin{array}{c}0 / 5 \\
(0 \%)\end{array}$ \\
\hline PKC $\zeta-$ & $\begin{array}{c}0 / 5 \\
(0 \%)\end{array}$ & $\begin{array}{c}0 / 5 \\
(0 \%)\end{array}$ & $\begin{array}{c}5 / 5 \\
(100 \%)\end{array}$ & $\begin{array}{c}5 / 5 \\
(100 \%)\end{array}$ \\
\hline$E R \alpha^{+}$ & $\begin{array}{c}0 / 5 \\
(0 \%)\end{array}$ & $\begin{array}{c}0 / 5 \\
(0 \%)\end{array}$ & $\begin{array}{c}5 / 5 \\
(100 \%)\end{array}$ & $\begin{array}{c}5 / 5 \\
(100 \%)\end{array}$ \\
\hline$E R \alpha-$ & $\begin{array}{c}5 / 5 \\
(100 \%)\end{array}$ & $\begin{array}{c}5 / 5 \\
(100 \%)\end{array}$ & $\begin{array}{c}0 / 5 \\
(0 \%)\end{array}$ & $\begin{array}{c}0 / 5 \\
(0 \%)\end{array}$ \\
\hline
\end{tabular}

b

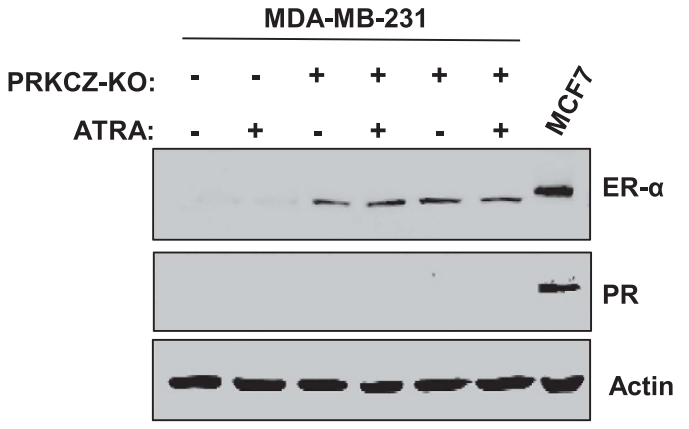

d
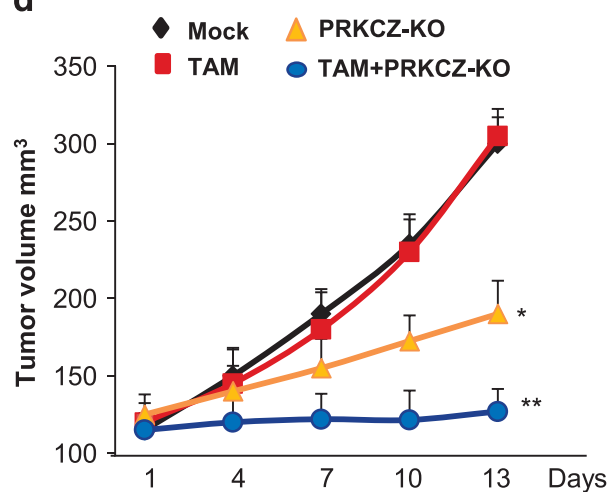

9 - Primary sphere Secondary sphere

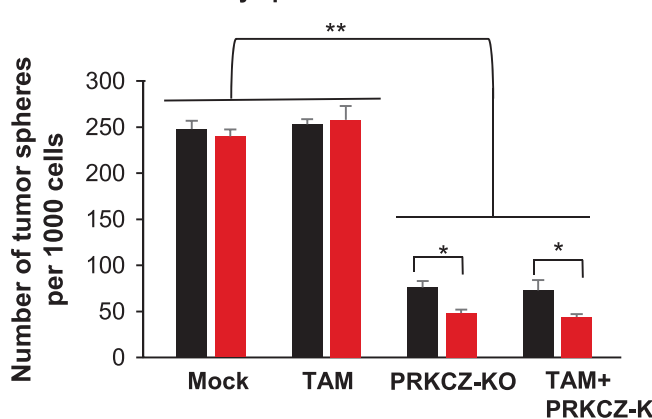

Figure 7. Inhibition of $\mathrm{PKC \zeta}$ directs breast CSCs to the luminal cell-like state and re-sensitization to TAM. (a, b) Protein expression levels of PKC $\zeta$, p-NUMB or ER $\alpha / P R$ in PRKCZ-KO or the control MDA-MB-231 breast cancer cells treated with $10^{-6} \mathrm{M}$ ATRA or vehicle for 24 h. (c) Extreme limiting dilution analysis showing the calculated CSC frequency by the xenograft tumor formation in the mammary fat pads of the 6-week-old female nude mice inoculated with the indicated inoculated number cells pre-treated with ATRA or vehicle for 7 days. (d) Growth curve of mammary xenograft tumors, (e) IHC staining images of PKC $\zeta$ and ER $\alpha$ expression in the xenograft tumor sections and (f) Fisher's exact correlation analysis showing a negative correlation of PKC $\zeta$ with ER $\alpha$ expression levels $(n=5$ animals/group, $P=0.00003,(-)$, negative-low staining; (+), strong-positive staining), and (g) the number of serially passaged tumor spheres (sphere size $>100 \mu \mathrm{m}$ ) per 1000 initially plated cells isolated from the xenograft tumors of the 6-week-old female nude mice that had been inoculated with $10^{5}$ PRKCZ-KO or control MDA-MB-231 breast cancer cells and then treated with TAM $(5 \mathrm{mg} / \mathrm{kg}$ i.p. every other day for 2 weeks) or vehicle when the tumors reached $100 \mathrm{~mm}^{3}$ ( $n=5$ animals per group, asterisk indicates $P<0.05$, double asterisk indicates $P<0.05$; scale bar: $50 \mu \mathrm{m}$ ). Comparable amount of control vector and vehicle were included as negative control (-) in each group. Error bars denote \pm s.d. 


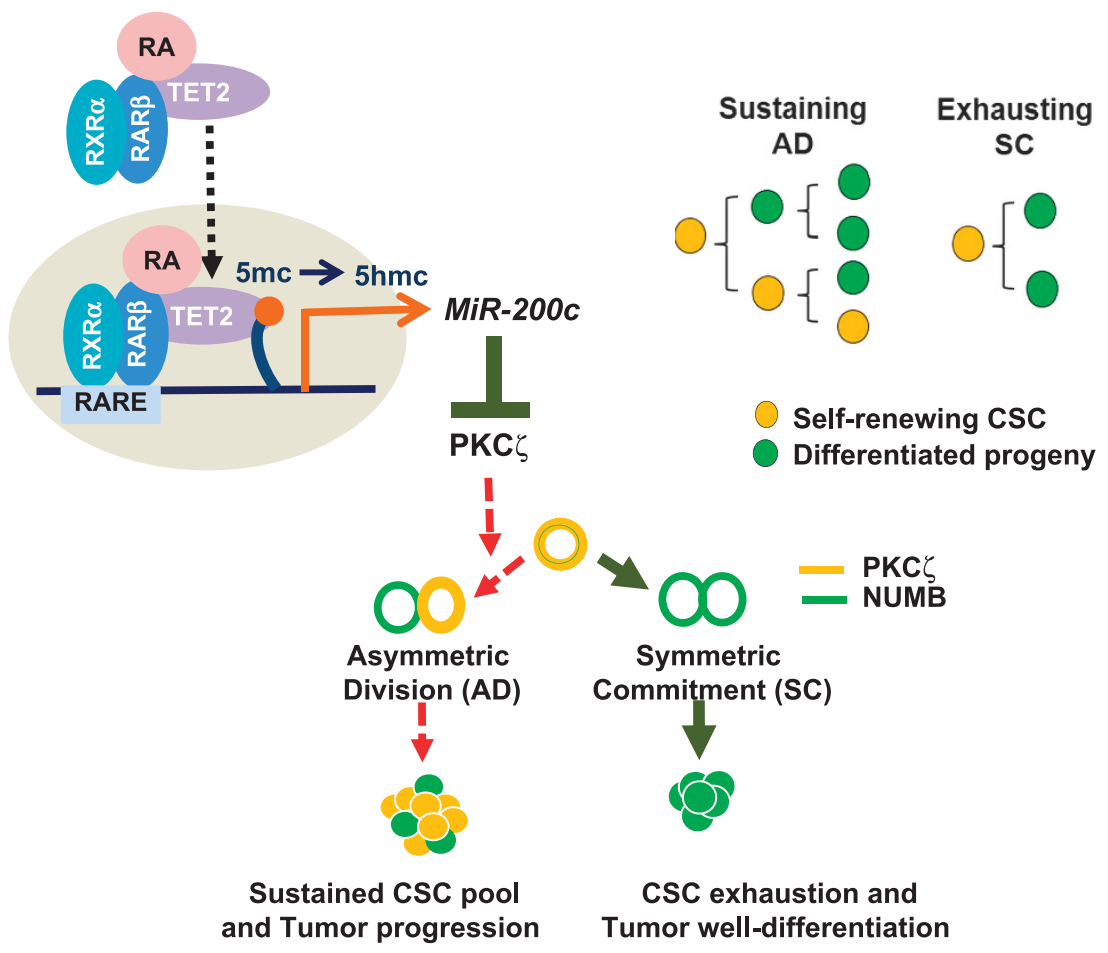

Figure 8. A proposed model illustrating ATRA directs breast cancer cell state changes to diminish the CSC pool through regulation of RAR $\beta$ TET2-miR-200c-PKC $\zeta$ pathway with schematic showing AD or SC cell fate decisions are made over accumulated CSC divisions to sustain or diminish the CSC pool.

width $\left.^{2}\right) / 2$. Four weeks after inoculation, tumors were collected for RNA/ protein extraction, sphere formation analysis following single-cell isolation, and also fixed in $10 \%$ neutral formalin for histological evaluation. The expected frequency of the tumorigenic CSCs was calculated using Extreme Limiting Dilution Analysis. ${ }^{32}$

\section{Statistical analysis}

All data were presented as means \pm the s.d. of the mean. Statistical calculations were performed with Microsoft Excel analysis tools. Differences between individual groups were analyzed by two-tailed paired $t$-test. $P$-values of $<0.05$ considered statistically significant. Chi-square analysis for tumor samples were performed as described previously. ${ }^{17}$

\section{CONFLICT OF INTEREST}

The authors declare no conflict of interest.

\section{ACKNOWLEDGEMENTS}

We thank the assistance of Dr Chao-Ching Chang as well as Dr Chi-Hong Chao with advice on animal and biochemical experiments, and Mr Chun-Chih Hsu with LC-MS/ MS analysis. This study was supported by Department of Defense Breast Cancer Breakthrough Award (W81XWH-15-1-0644 to C-J Chang), V foundation for Cancer Research Grant (V2015-019 to C-J Chang), and P30 CA023168 to the Purdue University Center for Cancer Research in support of the use of facilities.

\section{AUTHOR CONTRIBUTIONS}

M-JW and C-JC designed and conceived the study. M-JW and C-JC performed the experiments and wrote the manuscript. MRK and Y-SC helped with the biochemical experiments.

\section{REFERENCES}

1 Polyak K, Weinberg RA. Transitions between epithelial and mesenchymal states: acquisition of malignant and stem cell traits. Nat Rev Cancer 2009; 9: 265-273.
2 Reya T, Morrison SJ, Clarke MF, Weissman IL. Stem cells, cancer, and cancer stem cells. Nature 2001; 414: 105-111.

3 Visvader JE, Lindeman GJ. Cancer stem cells in solid tumours: accumulating evidence and unresolved questions. Nat Rev Cancer 2008; 8: 755-768.

4 Pece S, Tosoni D, Confalonieri S, Mazzarol G, Vecchi M, Ronzoni S et al. Biological and molecular heterogeneity of breast cancers correlates with their cancer stem cell content. Cell 2010; 140: 62-73.

5 Martin-Belmonte F, Perez-Moreno M. Epithelial cell polarity, stem cells and cancer. Nat Rev Cancer 2012; 12: 23-38.

6 Smith CA, Lau KM, Rahmani Z, Dho SE, Brothers G, She YM et al. aPKC-mediated phosphorylation regulates asymmetric membrane localization of the cell fate determinant Numb. EMBO J 2007; 26: 468-480.

7 Knoblich JA. Asymmetric cell division: recent developments and their implications for tumour biology. Nat Rev Mol Cell Biol 2010; 11: 849-860.

8 Garattini E, Gianni M, Terao M. Cytodifferentiation by retinoids, a novel therapeutic option in oncology: rational combinations with other therapeutic agents. Vitam Horm 2007; 75: 301-354.

9 Cuzick J, DeCensi A, Arun B, Brown PH, Castiglione M, Dunn B et al. Preventive therapy for breast cancer: a consensus statement. Lancet Oncol 2011; 12: 496-503.

10 Sutton LM, Warmuth MA, Petros WP, Winer EP. Pharmacokinetics and clinical impact of all-trans retinoic acid in metastatic breast cancer: a phase II trial. Cancer Chemother Pharmacol 1997; 40: 335-341.

11 Bryan M, Pulte ED, Toomey KC, Pliner L, Pavlick AC, Saunders T. A pilot phase II trial of all-trans retinoic acid (Vesanoid) and paclitaxel (Taxol) in patients with recurrent or metastatic breast cancer. Invest New Drugs 2011; 29: 1482-1487.

12 Veronesi U, De Palo G, Marubini E, Costa A, Formelli F, Mariani L et al. Randomized trial of fenretinide to prevent second breast malignancy in women with early breast cancer. J Natl Cancer Inst 1999; 91: 1847-1856.

13 Tang X-H, Gudas LJ. Retinoids, retinoic acid receptors, and cancer. Annu Rev Pathol Mech Dis 2011; 6: 345-364.

14 Ito S, D'Alessio AC, Taranova OV, Hong K, Sowers LC, Zhang Y. Role of Tet proteins in $5 \mathrm{mC}$ to $5 \mathrm{hmC}$ conversion, ES-cell self-renewal and inner cell mass specification. Nature 2010; 466: 1129-1133.

15 Song SJ, Poliseno L, Song MS, Ala U, Webster K, Ng C et al. MicroRNA-antagonism regulates breast cancer stemness and metastasis via TET-family-dependent chromatin remodeling. Cell 2013; 154: 311-324. 
16 Chen Q, Chen Y, Bian C, Fujiki R, Yu X. TET2 promotes histone O-GlcNAcylation during gene transcription. Nature 2013; 493: 561-564.

17 Chang C-J, Chao C-H, Xia W, Yang J-Y, Xiong Y, Li C-W et al. p53 regulates epithelial-mesenchymal transition and stem cell properties through modulating miRNAs. Nat Cell Biol 2011; 13: 317-323.

18 Shimono Y, Zabala M, Cho RW, Lobo N, Dalerba P, Qian D et al. Downregulation of miRNA-200c links breast cancer stem cells with normal stem cells. Cell 2009; 138: 592-603.

19 Chao C-H, Chang C-C, Wu M-J, Ko H-W, Wang D, Hung M-C et al. MicroRNA-205 signaling regulates mammary stem cell fate and tumorigenesis. J Clin Invest 2014; 124: 3093-3106.

20 Mani SA, Guo W, Liao M-J, Eaton EN, Ayyanan A, Zhou AY et al. The epithelialmesenchymal transition generates cells with properties of stem cells. Cell 2008; 133: 704-715.

21 Lee GY, Kenny PA, Lee EH, Bissell MJ. Three-dimensional culture models of normal and malignant breast epithelial cells. Nat Methods 2007; 4: 359-365.

22 Urtreger AJ, Kazanietz MG, Bal de Kier Joffé ED. Contribution of individual PKC isoforms to breast cancer progression. IUBMB Life 2012; 64: 18-26.

23 Sanjana NE, Shalem O, Zhang F. Improved vectors and genome-wide libraries for CRISPR screening. Nat Methods 2014; 11: 783-784.

24 Kumar BNP, Rajput S, Dey KK, Parekh A, Das S, Mazumdar A et al. Celecoxib alleviates tamoxifen-instigated angiogenic effects by ROS-dependent VEGF/ VEGFR2 autocrine signaling. BMC Cancer 2013; 13: 273.

25 Antal CE, Hudson AM, Kang E, Zanca C, Wirth C, Stephenson NL et al. Cancerassociated protein kinase $C$ mutations reveal kinase's role as tumor suppressor. Cell 2015; 160: 489-502.

26 Urtreger AJ, Grossoni VC, Falbo KB, Kazanietz MG, Bal de Kier Joffé ED. Atypical protein kinase C-zeta modulates clonogenicity, motility, and secretion of proteolytic enzymes in murine mammary cells. Mol Carcinogenesis 2005; 42: 29-39.
27 Castoria G, Migliaccio A, Di Domenico M, Lombardi M, de Falco A, Varricchio L et al. Role of atypical protein kinase $C$ in estradiol-triggered $\mathrm{G} 1 / \mathrm{S}$ progression of MCF-7 cells. Mol Cell Biol 2004; 24: 7643-7653.

28 Paul A, Danley M, Saha B, Tawfik O, Paul S. PKC promotes breast cancer invasion by regulating expression of E-cadherin and Zonula occludens-1 (ZO-1) via NFKBp65. Sci Rep 2015; 5: 12520.

29 Zhang F, Zhang X, Li M, Chen P, Zhang B, Guo H et al. mTOR complex component Rictor interacts with PKCzeta and regulates cancer cell metastasis. Cancer Res 2010; 70: 9360-9370.

30 Chang C-C, Wu M-J, Yang J-Y, Camarillo IG, Chang C-J. Leptin-STAT3-G9a signaling promotes obesity-mediated breast cancer progression. Cancer Res 2015; 75: 2375-2386.

31 Elstner E, Muller C, Koshizuka K, Williamson EA, Park D, Asou H et al. Ligands for peroxisome proliferator-activated receptorgamma and retinoic acid receptor inhibit growth and induce apoptosis of human breast cancer cells in vitro and in BNX mice. Proc Natl Acad Sci USA 1998; 95: 8806-8811.

$32 \mathrm{Hu}$ Y, Smyth GK. ELDA: extreme limiting dilution analysis for comparing depleted and enriched populations in stem cell and other assays. J Immunol Methods 2009; 347: 70-78.

(C) (1) This work is licensed under a Creative Commons Attributioncc) NonCommercial-ShareAlike 4.0 International License. The images or other third party material in this article are included in the article's Creative Commons license, unless indicated otherwise in the credit line; if the material is not included under the Creative Commons license, users will need to obtain permission from the license holder to reproduce the material. To view a copy of this license, visit http:// creativecommons.org/licenses/by-nc-sa/4.0/

(c) The Author(s) 2017

Supplementary Information accompanies this paper on the Oncogene website (http://www.nature.com/onc) 